\title{
\begin{tabular}{l|l} 
Mitraries & DSpace@MIT
\end{tabular}
}

\author{
MIT Open Access Articles
}

\section{Kardiovaskuläre Pharmakotherapie und koronare Revaskularisation bei terminaler Niereninsuffizienz}

The MIT Faculty has made this article openly available. Please share how this access benefits you. Your story matters.

Citation: Lauder, L. et al. "Kardiovaskuläre Pharmakotherapie und koronare Revaskularisation bei terminaler Niereninsuffizienz." Herz 44, 7 (August 2019): 611-629 (c) 2019 Springer Medizin Verlag $\mathrm{GmbH}$

As Published: https://doi.org/10.1007/s00059-019-04846-6

Publisher: Springer Science and Business Media LLC

Persistent URL: https://hdl.handle.net/1721.1/128615

Version: Author's final manuscript: final author's manuscript post peer review, without publisher's formatting or copy editing

Terms of Use: Article is made available in accordance with the publisher's policy and may be subject to US copyright law. Please refer to the publisher's site for terms of use. 


\title{
Kardiovaskuläre Pharmakotherapie und koronare Revaskularisation bei terminaler Niereninsuffizienz
}

\section{Cardiovascular drug therapy and coronary revascularization in end- stage renal disease}

\author{
L. Lauder ${ }^{1}$, S. Ewen ${ }^{1}$, I. E. Emrich ${ }^{1}$, M. Böhm¹, F. Mahfoud ${ }^{1,2}$ \\ ${ }^{1}$ Klinik für Innere Medizin III, Kardiologie, Angiologie und Internistische Intensivmedizin, Universitätsklinikum des \\ Saarlandes und Medizinische Fakultät der Universität des Saarlandes, Homburg/Saar, Deutschland \\ 2 Institute for Medical Engineering and Science, Massachusetts Institute of Technology, Cambridge, MA, USA
}

\section{Korrespondenzadresse}

Dr. med. Lucas Lauder

Klinik für Innere Medizin III,

Kardiologie, Angiologie und internistische Intensivmedizin

Universitätsklinikum des Saarlandes

Kirrberger Str. 100, 66421 Homburg/Saar

Fon: $+496841 / 1615911$

Fax: +49 6841/16 15910

Lucas.Lauder@uks.eu

\section{Zusammenfassung}

Herz und Nieren sind in ihrer physiologischen Interaktion eng miteinander verbunden.

Kardiovaskuläre Erkrankungen sind die häufigste Todesursache bei Patienten mit chronischer Nierenerkrankung, wobei diese wiederum das Auftreten und Fortschreiten kardiovaskulärer Erkrankungen begünstigen kann. Die Therapie der koronaren Herzkrankheit und chronischen Herzinsuffizienz unterscheidet sich bei Patienten mit einer milden Nierenfunktionseinschränkung (eGFR $>60 \mathrm{ml} / \mathrm{min} / 1,73 \mathrm{~m}^{2}$ ) nicht wesentlich von der Therapie nierengesunder Patienten. Da Patienten mit einer fortgeschrittenen Niereninsuffizienz aus den Zulassungsstudien meist ausgeschlossen wurden, basieren viele Therapieempfehlungen in dieser Patientengruppe auf Beobachtungsstudien, Subgruppenund Metaanalysen oder pathophysiologischen Erwägungen, die nicht durch kontrollierte Studien gestützt sind. Daher werden prospektive, randomisierte Studien zur Therapie der koronaren Herzkrankheit und Herzinsuffizienz benötigt, die Patienten mit fortgeschrittener Nierenfunktionseinschränkung gezielt untersuchen.

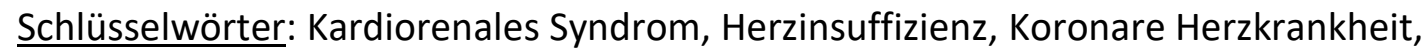
Niereninsuffizienz 


\begin{abstract}
There is a close relationship between the kidneys and the heart. Cardiovascular disease is the most prevalent cause of death in patients with chronic kidney disease (CKD), whereas CKD may directly accelerate the progression of cardiovascular disease and is considered to be cardiovascular risk factor. In patients with mild CKD (eGFR $>60 \mathrm{ml} / \mathrm{min} / 1.73 \mathrm{~m}^{2}$ ) treatment of coronary artery disease and chronic heart failure is not different from patients with preserved renal function. However, most pivotal trials have systematically excluded patients with end-stage renal disease. Due to the scarcity of data in these patients, treatment recommendations are mainly based on observational studies as well as post-hoc and meta-analyses or pathophysiological considerations but not trial evidence. Therefore, studies on the management of heart failure and coronary artery disease should specifically focus on the growing number of patients with CKD.
\end{abstract}

Key words: Cardio-renal syndrome, heart failure, coronary heart disease, chronic kidney disease

\title{
Abkürzungsverzeichnis
}

ARNI = Angiotensin-Rezeptor-Neprilysin-Inhibitor

$\mathrm{CKD}=$ chronische Niereninsuffizienz (engl. chronic kidney disease)

eGFR = geschätzte glomeruläre Filtrationsrate (engl. estimated glomerular filtration rate)

$F F R=$ fraktionelle Flussreserve

HFrEF/HFmrEF/HFpEF = Herzinsuffizienz mit reduzierter/mittelgradig

eingeschränkter/erhaltener Ejektionsfraktion

KDIGO = Kidney Disease: Improving Global Outcomes

$\mathrm{KHK}=$ Koronare Herzkrankheit

MACCE = schwere kardiale und zerebrovaskuläre Ereignisse (engl. major adverse cardiac and cardiovascular events)

MRA = Mineralokortikoid-Rezeptorantagonist

NKCC $=$ Natrium-Kalium-Chlorid-Cotransporter

LVEF = Linksventrikuläre Ejektionsfraktion

RAAS = Renin-Angiotensin-Aldosteron-System

\section{Lernziel}

Nach Lektüre dieser Lerneinheit sollten Ihnen die Besonderheiten der Therapie der stabilen koronaren Herzkrankheit und der chronischen Herzinsuffizienz bei Patienten mit fortgeschrittener chronischer Nierenerkrankung bekannt sein. 


\section{Einleitung}

Das Herz und die Nieren sind in ihrer physiologischen Interaktion vielfältig miteinander verbunden (Abb. 1) [1]. Kardiovaskuläre Erkrankungen sind die häufigste Ursache für Morbidität und Sterblichkeit bei Patienten mit chronischer Niereninsuffizienz (CKD) [2]. Die CKD begünstigt das Fortschreiten kardiovaskulärer Erkrankungen und ist als unabhängiger Risikofaktor für kardiovaskuläre Ereignisse anerkannt [2]. Die Unterteilung der CKD wird von der KDIGO (Kidney Disease: Improving Global Outcomes) als eine $\geq 3$ Monate anhaltende strukturelle oder funktionelle Veränderung der Nieren definiert, deren

Schweregradeinteilung anhand der geschätzten glomerulären Filtrationsrate (eGFR) und der Albuminurie erfolgt (Abb. 2) [3]. Das kardiorenale Syndrom beschreibt einen Krankheitskomplex bei dem eine Schädigung von Herz oder Niere sekundär zur Dysfunktion des jeweils anderen Organs führt. Anhand der Genese (primär kardial oder renal) und des Verlaufs (akut oder chronisch) wird das kardiorenale Syndrom in fünf Subtypen (Tab. 1) unterteilt, die nicht immer eindeutig zu differenzieren sind [4].

\section{Stabile koronare Herzkrankheit (КНK)}

\section{KHK und CKD}

Die CKD wird als wichtiger Risikofaktor für die Entstehung einer Atherosklerose angesehen und ist eine der häufigsten Komorbiditäten der KHK [5]. Eine Abnahme der eGFR ist mit einem zunehmenden Risiko für kardiovaskuläre Sterblichkeit assoziiert [6]. Die Komplikationen der KHK (Myokardinfarkt, Herzinsuffizienz) stellen die häufigsten Todesursachen von Patienten mit CKD dar [5].

\section{Diagnostische Besonderheiten der KHK bei CKD}

Bei Patienten mit CKD sind pektanginöse Beschwerden seltener und die Prävalenz stummer Myokardischämie häufiger [7]. Zudem sind die Sensitivität und Spezifität des klinischen Symptoms der Angina pectoris (Sensitivität 65\%, Spezifität 66\%), des Ruhe-EKGs (Sensitivität $67 \%$, Spezifität 52\%) oder der Perfusionsszintigraphie (Sensitivität 74\%, Spezifität 70\%) bei Patienten mit terminaler Niereninsuffizienz deutlich schlechter als bei nierengesunden Patienten [8, 9]. Auch die Aussagekraft der invasiven Ischämiediagnostik kann bei Patienten mit CKD reduziert sein. So konnte gezeigt werden, dass die Validität der intrakoronar gemessenen fraktionellen Flussreserve (FFR) bei Patienten mit CKD (Kreatinin-Clearance $\leq 45$ $\mathrm{mL} / \mathrm{min}$ ) eingeschränkt ist, da der FFR-Wert bei Patienten mit CKD signifikant seltener pathologisch (FFR $\leq 0,8)$ war als in der Kontrollgruppe ( $27 \%$ vs. $41 \%, p<0,01)$ [10]. Daher sollte bei der Wahl der Methode zur Diagnostik einer KHK immer auch die Nierenfunktion berücksichtigt werden. Hierbei sollte die Kontrastmittelexposition auf ein Minimum reduziert werden, um eine weitere Funktionsverschlechterung der Nieren zu vermeiden [11].

\section{Medikamentöse Therapie der stabilen KHK}

Die medikamentöse Therapie der stabilen KHK soll eine Symptomkontrolle erzielen, das Fortschreiten der KHK verlangsamen und das Auftreten kardiovaskulärer Ereignisse reduzieren $[12,13]$. Bislang gibt es jedoch keine randomisierten, kontrollierten Studien, die medikamentöse Therapiestrategien der KHK bei Patienten mit fortgeschrittener Niereninsuffizienz untersucht haben. Aktuelle Leitlinien empfehlen den Einsatz von Betablockern und Kalziumkanalblockern als Erstlinientherapie bei stabiler KHK [13]. Eine 
Netzwerk-Analyse mit 46 Studien konnte den klinischen Nutzen einer Kombinationstherapie aus Kalziumkanal- und Betablockern auf die Frequenz von Angina pectoris und Nitratbedarf zeigen [14]. Neben Kalziumkanal- und Betablockern können auch Nitrate bei Patienten mit CKD eingesetzt werden, da diese vor allem hepatisch metabolisiert werden und daher weniger kumulieren. Werden initial Kalziumkanalblocker vom Dihydropyridin-Typ oder Nitrate verwendet, kann eine Kombinationstherapie mit Betablockern sinnvoll sein, um eine reflektorische Tachykardie zu verhindern und die anti-anginöse Wirkung zu verstärken [12, 13]. Eine Kombination von Betablockern mit Verapamil oder Diltiazem sollte aufgrund des Risikos einer Bradykardie oder eines AV-Blocks vermieden werden [13]. Die Piperazinderivate Ranolazin und Trimetazidin (in Deutschland nicht verfügbar) sollten bei Patienten mit CKD nur vorsichtig eingesetzt werden, da die Plasmaspiegel aufgrund der renalen Akkumulation deutlich ansteigen und interindividuell stark variieren können [12]. Die Bioverfügbarkeit von Ranolazin (Area under the curve der Konzentrations-Zeit-Kurve) war bei Patienten mit einer Kreatinin-Clearance $<30 \mathrm{ml} / \mathrm{min} / 1,73 \mathrm{~m}^{2} \mathrm{im}$ Vergleich zu Nierengesunden beispielsweise um das 1,97-fache (90\%-Konfidenzintervall (Cl) 1,23-3,16) erhöht [15]. Ivabradin, ein If-Kanal-Blocker, führt durch einen isoliert negativ chronotropen Effekt zu einer Verminderung des myokardialen Sauerstoffverbrauchs und kann so im Vergleich zu Placebo die Belastungstoleranz verbessern sowie die Ausprägung der AnginaSymptomatik, die Anzahl der Angina pectoris-Anfälle und den Verbrauch kurzwirksamer Nitrate bei Patienten mit stabiler KHK reduzieren [16]. In der SIGNIFY-Studie führte Ivabradin im Vergleich zu Placebo bei 19.102 Patienten mit KHK und ohne klinische Herzinsuffizienz unter optimaler Basistherapie zu keiner Reduktion des primären Endpunkts aus kardiovaskulärer Sterblichkeit und nicht-tödlichem Myokardinfarkt (HR 1,08; 95\%-Cl 0,96$1,20)$ [17]. Da Ivabradin fast vollständig hepatisch metabolisiert wird und die renale Clearance nur etwa 20\% der Ivabradin-Elimination ausmacht, gilt die Verwendung von Ivabradin auch bei CKD als sicher [18].

\section{Thrombozytenaggregationshemmung}

Bei Patienten mit CKD ist sowohl das Risiko für thromboembolische Ereignisse als auch für Blutungskomplikationen im Vergleich zu Patienten mit erhaltener Nierenfunktion erhöht [19, 20]. Für die gesteigerte Thrombogenität bei CKD ist unter anderem eine Dichtezunahme von Thrombozytenclotts bei abnehmender Nierenfunktion verantwortlich [21]. Diese gilt bei Hämodialyse-Patienten als unabhängiger Risikofaktor für die Gesamt- und kardiovaskuläre Sterblichkeit [21]. Da die Evidenz für Acetylsalicylsäure und P2Y12-Rezeptorantagonisten zum Einsatz bei Patienten mit CKD limitiert ist, werden durch die Leitlinien keine gesonderten Therapieempfehlungen ausgesprochen [13]. Eine Metaanalyse der Cochrane Collaboration mit insgesamt 27.139 niereninsuffizienten Patienten (definiert als eGFR $<60$ $\mathrm{ml} / \mathrm{min} / 1,73 \mathrm{~m}^{2}$, Albuminurie oder Serumkreatinin $>1,36 \mathrm{mg} / \mathrm{dl}$ ), einschließlich 4.820 Dialyse-Patienten, konnte zeigen, dass Thrombozytenaggregationshemmer das Risiko für einen Myokardinfarkt um 13\% reduzieren können (relatives Risiko (RR) 0,87; 95\%-Cl 0,76$0,99)$ [19]. Die kardiovaskuläre und die Gesamtsterblichkeit wurden ebenso wie das Schlaganfallrisiko durch den Einsatz von Thrombozytenaggregationshemmer in dieser Patientengruppe nicht beeinflusst [19]. Erwartungsgemäß war das Blutungsrisiko unter Therapie mit Thrombozytenaggregationshemmern erhöht [19]. In einer kleinen Studie war die residuelle Thrombozytenreaktivität unter Clopidogrel, als Zeichen einer eingeschränkten antithrombotischen Wirksamkeit, bei terminaler CKD hoch [22]. Die Angst vor Blutungskomplikationen und die fraglich eingeschränkte Wirksamkeit tragen möglicherweise dazu bei, dass bei niereninsuffizienten Patienten häufig keine leitliniengerechte 
Thrombozytenaggregationshemmung initiiert oder die empfohlene Dauer einer dualen Plättchenhemmung nicht eingehalten wird [23]. Einige Besonderheiten zum Einsatz von Thrombozytenaggregationshemmern bei CKD sind in Tab. 2 dargestellt [24].

\section{Lipidsenkende Therapie}

Statine als lipidsenkende Therapie sind auch bei Patienten mit CKD essentieller Bestandteil der kardiovaskulären Primär- und Sekundärprävention $[25,26]$. Allerdings untersuchten nur wenige Studien die Wirksamkeit und Sicherheit von Statinen bei Patienten mit CKD. Die Kombination aus Simvastatin 20 mg plus Ezetimib 10 mg konnte in der SHARP-Studie bei 9.270 Patienten mit CKD (Serum-Kreatinin >1,7 mg/dl bei Männern, >1,5 mg/dl bei Frauen) die Anzahl schwerer atherosklerotischer Ereignisse um 17\% reduzieren (RR 0,83; 95\%-Cl $0,74-0,94)$ [27]. Da das Risiko einer myokardialen Ischämie mit Todesfolge oder eines nichttödlichen Myokardinfarkts bei Patienten mit CKD (>50 Jahre) in einer Untersuchung des Alberta Kidney Disease Network stadienunabhängig mehr als 10\% für die folgenden 10 Jahre betrug [28], empfiehlt die KDIGO-Leitlinie für alle Patienten $>50$ Jahren eine lipidsenkende Therapie mit einem Statin oder Simvastatin plus Ezetimib (Klasse IA für CKD G3a-G5 und Klasse IB für CKD G1-G2) [26]. Die ESC/EAS-Leitlinien zur Diagnostik und Therapie von Dyslipidämien empfehlen bei allen Patienten mit nicht-dialysepflichtiger CKD KDIGO GFRStadium G3-G5 eine Therapie mit Statinen oder einer Statin/Ezetimib-Kombination, wobei hepatisch eliminierte Substanzen (Fluvastatin, Atorvastatin, Pitavastatin und Ezetimib) zu bevorzugen sind, um einen LDL-Zielwert $<70 \mathrm{mg} / \mathrm{dl}$ zu erreichen [25]. Bei dialysepflichtigen Patienten konnte in drei randomisierten, kontrollierten Studien weder für Simvastatin plus Ezetimib (SHARP [27]), noch Atorvastatin (Die Deutsche Diabetes Dialyse [4D]-Studie [29]) oder Rosuvastatin (AURORA [30]), trotz einer suffizienten Lipidsenkung, eine Reduktion von kardiovaskulären Ereignissen oder der Sterblichkeit gezeigt werden. Daher empfehlen sowohl die KDIGO als auch die ESC/EAS Leitlinie, dass eine bestehende Statin-Therapie bei dialysepflichtigen Patienten fortgeführt werden kann (Klasse $2 \mathrm{C}$ ), aber nicht neu initiiert werden sollte (Klasse 2B) [25, 26]. Da die Evidenz für Statine besser ist als für Fibrate, empfiehlt die KDIGO Statine gegenüber Fibraten bei Patienten mit CKD zu bevorzugen [25, 26].

\section{Revaskularisationsstrategien}

Bei niereninsuffizienten Patienten werden häufiger Hauptstammstenosen und koronare Mehrgefäßerkrankungen mit komplexen Läsionen (ausgeprägte Kalzifikation, kleine Gefäße und langstreckige Stenosen) beobachtet, wobei die Komplexität der Läsionen mit abnehmender Nierenfunktion zunimmt [31]. Des Weiteren haben Patienten, die einer Koronarrevaskularisation (egal ob perkutan oder chirurgisch) unterzogen werden, ein erhöhtes Sterblichkeitsrisiko, häufiger Blutungskomplikationen sowie ein höheres ReStenose-Risiko [32]. Obgleich eine Vielzahl von Studien die Vorteile einer Koronarrevaskularisation mittels $\mathrm{PCl}$ und operativer Myokardrevaskularisation (CABG) im Vergleich zu einer konservativen Therapie bei Patienten mit CKD dokumentieren konnte, werden Patienten mit CKD insgesamt seltener einer Koronarrevaskularisation zugeführt als nierengesunde Patienten [9, 33]. Im vergangenen Jahr wurden Subgruppenanalysen der prospektiven, randomisierten SYNTAX- [34] und EXCEL-Studien [35] veröffentlicht. In beiden Studien wurden Patienten mit einer Mehrgefäß-KHK (zum Teil mit Hauptstammbeteiligung) entweder durch CABG oder mit Drug-eluting Stents (DES) PCI behandelt. Die SYNTAX-Studie zeigte, dass die Koronarrevaskularisation bei Patienten mit CKD (eGFR $<60 \mathrm{ml} / \mathrm{min} / 1,73 \mathrm{~m}^{2}$ ) 
im Vergleich zu nierengesunden Patienten mit einer deutlich höheren Mortalität nach fünf Jahren assoziiert war (PCl: 26,7 vs. 10,8\%, p<0,001; CABG: $21,2 \%$ vs. $10,6 \%, p=0,005$ ) [34]. Die Rate schwerer kardialer und zerebrovaskulärer Ereignisse (MACCE) war nach $\mathrm{PCl}$, insbesondere aufgrund des erhöhten Risikos für eine erneute Revaskularisation, höher als nach einer Bypass-Operation (42,1\% vs. 31,5\%, p=0,019) [34]. Die EXCEL-Studie konnte bei Patienten mit Hauptstammstenose und CKD keinen Unterschied hinsichtlich der Häufigkeit des kombinierten primären Endpunkts aus Tod, Myokardinfarkt und Schlaganfall nach drei Jahren zwischen der PCl- und der CABG-Gruppe zeigen [35]. In der PCl-Gruppe war der kombinierte primäre Endpunkt nach 30 Tagen signifikant seltener, die Krankenhausaufenthaltsdauer kürzer und die Wahrscheinlichkeit eines akuten Nierenversagens geringer. Die Mortalitätsrate nach drei Jahren, vor allem bedingt durch eine erhöhte Rate nicht-kardialer Todesfälle, war in der PCl-Gruppe höher $(5,4 \%$ vs. 1,1\%, p=0,02) [35]. In einer Metaanalyse an 29.246 Patienten mit koronarer Mehrgefäßerkrankung und CKD war die DES-PCI im Vergleich zur Bypass-Operation mit einem erhöhten Risiko für Langzeitsterblichkeit, Myokardinfarkt, erneuter Revaskularisation und MACCE assoziiert, wohingegen die kurzfristige Sterblichkeit und das Risiko zerebrovaskulärer Ereignisse nach $\mathrm{PCl}$ geringer waren [36]. Die Entscheidung für eine $\mathrm{PCl}$ oder die CABG-Operation sollte daher unter Berücksichtigung der Komorbiditäten, der Koronarmorphologie, der Möglichkeit einer kompletten Revaskularisation und unter Einbeziehung der Patientenwunsches individuell durch das Herz-Team getroffen werden.

\section{Herzinsuffizienz}

Die chronische Herzinsuffizienz ist ein klinisches Syndrom, das durch Symptome (z.B. Dyspnoe) und klinische Zeichen (z.B. Jugularvenenstauung, periphere Ödeme) gekennzeichnet ist [37]. Das Risiko eine Herzinsuffizienz zu entwickeln, ist schon bei einer milden (eGFR 60-89 ml/min/1,73 $\mathrm{m}^{2}$ ) bis moderaten (eGFR 30-59 ml/min/1,73 $\mathrm{m}^{2}$ ) CKD erhöht und steigt mit abnehmender Nierenfunktion weiter an [38]. Bei dialysepflichtigen Patienten ist das Risiko im Vergleich zur Normalbevölkerung um das zwölf- bis 36-fache erhöht [39]. Umgekehrt ist eine Herzinsuffizienz mit einem Fortschreiten der CKD und einem häufigeren Auftreten von akutem Nierenversagen assoziiert [40, 41]. Eine Albuminurie ist sowohl bei Herzinsuffizienz mit reduzierter [42] als auch mit erhaltener systolischer LVFunktion [43] mit einer ungünstigeren Prognose assoziiert.

\section{Herzinsuffizienztherapie bei Niereninsuffizienz}

Bislang konnte keine Therapie eine überzeugende Senkung der Morbidität und Sterblichkeit bei Patienten mit Herzinsuffizienz und erhaltener linksventrikulärer Funktion (HFpEF; LVEF 250\%) sowie Herzinsuffizienz mit mäßiggradig eingeschränkter (HFmrEF, LVEF 40-49\%) aufzeigen [37]. Daher wird im Folgenden die Therapie der Herzinsuffizienz mit reduzierter Ejektionsfraktion (HFrEF; LVEF <40\%) und begleitender CKD diskutiert. Bei Patienten mit einer reduzierten systolischen Pumpfunktion und einer CKD bis zu einem KDIGO GFRStadium G3b (eGFR $\geq 30 \mathrm{ml} / \mathrm{min} / 1,73 \mathrm{~m}^{2}$ ) unterscheidet sich die Herzinsuffizienztherapie nicht wesentlich von der Therapie bei Nierengesunden. Abb. 3 zeigt die Therapie der HFrEF bei Patienten mit einer fortgeschrittenen Niereninsuffizienz (eGFR $<30 \mathrm{ml} / \mathrm{min} / 1,73 \mathrm{~m}^{2}$ ). Wird ein Patient erstmals aufgrund einer akut dekompensierten HFrEF hospitalisiert, kann nach einer ausreichenden Stabilisierung direkt eine RAAS-Inhibition mittels ARNI begonnen werden, um bis zum Zeitpunkt der Krankenhausentlassung eine möglichst optimale Herzinsuffizienztherapie zu erzielen $[44,45]$. 


\section{Diuretika}

Der Verlauf einer chronischen Herzinsuffizienz ist durch rezidivierende kardiale Dekompensationen charakterisiert [46, 47]. Obwohl für Diuretika keine prospektiven, randomisierten Studien zur Prognosebesserung durchgeführt wurden, sind sie zur Symptomlinderung und zur Verbesserung der Belastungsfähigkeit essentiell und durch die Leitlinien ausdrücklich empfohlen (Klasse 1B) [37]. Hinweise für die prognostische Bedeutung von Schleifen- und Thiaziddiuretika finden sich in Metaanalysen [48, 49]. Dort konnte im Vergleich zu Placebo eine Reduktion der Sterblichkeit und der HerzinsuffizienzProgression gezeigt werden $[48,49]$. In der Herzinsuffizienztherapie kommen insbesondere Schleifendiuretika zum Einsatz [50]. Während die Blockade des Natrium-Kalium-ChloridCotransporters (NKCC)-2 im aufsteigenden Teil der Henle-Schleife für den Großteil des natriuretischen Effekts verantwortlich ist, steigert die Hemmung des NKCC1 im extraglomerulärem Mesangium die Reninsekretion [50]. Die orale Einnahme von Furosemid ist durch eine kurze Halbwertszeit von 2,7 Stunden und eine ausgeprägte Variabilität der Bioverfügbarkeit von 10-90\% gekennzeichnet [50]. Torasemid ist aufgrund seiner konstanten Bioverfügbarkeit $>90 \%$ und seiner längeren Halbwertszeit von etwa 6 Stunden möglicherweise besser für die Therapie von ambulanten herzinsuffizienten Patienten geeignet [50]. Diese Fragestellung wird aktuell in der großen, randomisierten TRANSFORMHF-Studie (NCT03296813) untersucht. Der Einsatz von Schleifendiuretika kann allerdings aufgrund seiner systemischen und renalen hämodynamischen Effekte sowie durch die Aktivierung des RAAS zu einer weiteren Verschlechterung der Nierenfunktion führen, die jedoch nicht mit einer dauerhaften Prognoseverschlechterung einhergehen muss [51, 52]. In der CARRESS-HF-Studie wurde die veno-venöse Ultrafiltration unter anderem als Alternative zur Diuretikatherapie untersucht [53]. Der Gewichtsverlust war unter einer pharmakologischen Stufentherapie aus intravenösen Schleifendiuretika mit und ohne Thiaziddiuretikum und einer Ultrafiltration vergleichbar [53]. Allerdings kam es bei Patienten, die mittels Ultrafiltration behandelt wurden, zu einem Anstieg des Serumkreatinins und vermehrt zu schweren unerwünschten Ereignissen [53]. Eine CKD begünstigt durch verschiedene pharmakokinetische und pharmakodynamische Mechanismen das Auftreten einer sogenannten Diuretika-Resistenz. So werden Schleifendiuretika unter anderem schlechter durch die proximalen Tubuluszellen sezerniert und weniger Natrium wird in den Primärharn filtriert [4]. Bei akut kardial dekompensierten Patienten mit einem hohen Risiko für eine Diuretika-Resistenz konnte die kontinuierliche Furosemid-Gabe die Stauungszeichen schneller reduzieren als eine Bolusgabe [54].

Außerdem kann durch den kombinierten Einsatz eines Schleifendiuretikums mit einem distal wirkenden Thiaziddiuretikum die distale (kompensatorisch erhöhte) Natriumrückresorption im Sinne einer sequentiellen Nephronblockade gehemmt und somit die Wirkung der Schleifendiuretika potenziert werden [4]. Eine Monotherapie mit Thiaziddiuretika ist bei Patienten mit einer eGFR $<30 \mathrm{~mL} / \mathrm{min} / 1,73 \mathrm{~m}^{2}$ eingeschränkt wirksam und sollte deshalb nicht durchgeführt werden.

\section{Betablocker}

Betablocker konnten in mehreren randomisierten, kontrollierten Studien bei Patienten mit HFrEF die Symptomatik und die LVEF verbessern sowie die Hospitalisierungsrate und die Sterblichkeit reduzieren [4]. Zu den Betablockern, für die eine Mortalitätsreduktion nachgewiesen wurde, zählen unter anderem Metoprolol (MERIT-HF [55]), Bisoprolol (CIBIS-II [56]), Carvedilol (US Carvedilol Programme [57]) und Nebivolol (SENIORS [58]). Keine der 
großen randomisierten Studien hatte prospektiv den Nutzen von Betablockern bei Patienten mit CKD untersucht (Tab. 3), sodass die Therapieempfehlungen vor allem auf post-hoc und Metaanalysen basieren [59]. Eine Subgruppenanalyse der MERIT-HF-Studie, in der 493 Patienten mit einer eGFR $<45 \mathrm{ml} / \mathrm{min} / 1,73 \mathrm{~m}^{2}$ (MDRD-Formel) und einer symptomatischen HFrEF entweder mit Metoprolol oder Placebo behandelt wurden, zeigte eine Reduktion des relativen Risikos für den kombinierten Endpunkt aus Herzinsuffizienzhospitalisierung und Gesamtsterblichkeit von 56\% [60]. Eine Metaanalyse aus sechs randomisierten, kontrollierten Studien hat die Wirkung der Betablocker bei insgesamt 5.792 Patienten mit CKD im KIDGO GFR-Stadium G3-5 untersucht [61]. Im Vergleich zu Placebo, reduzierten Betablocker die Gesamtsterblichkeit (RR 0,72; 95\%-Cl 0,60-0,80) und kardiovaskuläre Sterblichkeit (RR 0,66; 95\%-Cl 0,49-0,89), wobei allerdings auch das Risiko für eine Hypotonie (RR 5,08; 95\%-Cl 3,48-7,41) oder Bradykardie (RR 4,92; 95\%-Cl 3,20-7,55) deutlich erhöht war [61].

Bei Patienten mit fortgeschrittener CKD sollte auf Betablocker (z.B. Sotalol, Atenolol, Nadolol), die überwiegend renal ausgeschieden werden, verzichtet werden [62]. Eine kleinere, randomisierte Studie ( $\mathrm{N}=114)$ untersuchte die Wirksamkeit und Sicherheit von Betablockern bei Patienten mit dialysepflichtiger CKD und HFrEF. Carvedilol reduzierte in diesem Kollektiv sowohl die kardiovaskuläre Sterblichkeit (HR 0,32; 95\%-Cl 0,18-0,57) als auch die Anzahl der Hospitalisierungen (HR 0,44; 95\%-Cl 0,25-0,77) [63]. In einer retrospektiven Kohortenstudie war die Therapie mit gut dialysierbaren (z.B. Metoprolol) im Vergleich zu eingeschränkt dialysierbaren Betablockern (z.B. Bisoprolol) bei Patienten über 66 Jahren mit einer höheren 180-Tage-Sterblichkeit assoziiert (RR 1,4;95\%-Cl 1,1-1,8), sodass bei Patienten mit terminaler CKD Betablocker mit einer geringeren Dialysierbarkeit möglicherweise zu bevorzugen sind [64].

\section{ACE-Hemmer/Angiotensin-Rezeptorblocker}

Patienten mit fortgeschrittener CKD wurden aus den meisten Zulassungsstudien für ACEHemmer (Tab. 4) und Angiotensin-Rezeptorblocker (Tab. 5) bei HFrEF ausgeschlossen. In der SOLVD-Studie konnte bei Patienten mit einer CKD (eGFR $<60 \mathrm{ml} / \mathrm{min} / 1,73 \mathrm{~m}^{2}$ ) die Anzahl der Hospitalisierungen aufgrund kardiovaskulärer Ereignisse durch die Therapie mit Enalapril signifikant reduziert werden [65]. Die kardiovaskuläre, die Herzinsuffizienz- sowie die Gesamtsterblichkeit unterschieden sich nicht signifikant zwischen Patienten in der Enalaprilund Placebo-Gruppe [65]. In der CONSENSUS-Studie nahm bei $24 \%$ der Patienten, die mit Enalapril behandelt wurden, das Serum-Kreatinin im Verlauf ab. Bei 11\% der Patienten verdoppelte sich das Serum-Kreatinin, meist jedoch nur vorrübergehend während der ersten Wochen nach Therapiebeginn [66]. Im Rahmen des schwedischen Herzinsuffizienzregisters wurden insgesamt 2.410 Patienten mit HFrEF und fortgeschrittener Niereninsuffizienz (Serum-Kreatinin $>2,5 \mathrm{mg} / \mathrm{dl}$ oder Kreatinin-Clearance $<30 \mathrm{ml} / \mathrm{min}$ ) mit oder ohne RASInhibitor untersucht [67]. Mittels der Propensity-Score-Methode wurden je 602 Patienten mit und ohne AT1-Rezeptorblocker oder ACE-Hemmer miteinander verglichen [67]. Bei Patienten mit RAS-Inhibition war im Vergleich zu Patienten ohne RAS-Inhibition die Gesamtsterblichkeit nach einem Jahr signifikant niedriger (HR 0,76; 95\%-Cl 0,67-0,86) [67]. Zurzeit wird in der prospektiven STOP-ACEi-Studie (ISRCTN62869767) untersucht, ob das Absetzen von ACE-Hemmern oder Angiotensin-Rezeptorblockern bei Patienten in den KDIGO GFR-Stadien G4-5 die Nierenfunktion stabilisieren oder sogar verbessern kann.

\section{Angiotensin-Rezeptor-Neprilysin-Inhibitor (ARNI)}


In der PARADIGM-HF-Studie, war bei Patienten mit HFrEF die Therapie mit Valsartan/Sacubitril, einem Angiotensin-Rezeptor-Neprilysin-Inhibitor (ARNI), im Vergleich zu Enalapril mit einer Reduktion der Gesamtsterblichkeit (-16\%), der kardiovaskulären Mortalität (-20\%), der Herzinsuffizienzhospitalisierungen (-21\%) und einer Verbesserung der Lebensqualität (Verbesserung des Kansas City Cardiomyopathy Questionnaire (KCCQ), +64\%) assoziiert [68]. Schwere Hyperkaliämien (Kalium $>6 \mathrm{mmol} / \mathrm{l}$ ) traten unter Sacubitril/Valsartan im Vergleich zu Enalapril seltener auf (4,3\% vs. 5,6\%; $p=0,007)$ [69]. Zudem fiel die eGFR unter Sacubitril/Valsartan im Vergleich zur Kontrollgruppe langsamer ab (-1,61 (95\%-Cl 1,77-(-1,44)) $\mathrm{ml} / \mathrm{min} / 1,73 \mathrm{~m}^{2}$ vs. $\left.-2,04(95 \%-\mathrm{Cl}-2,21-(-1,88)) \mathrm{ml} / \mathrm{min} / 1,73 \mathrm{~m}^{2}\right)$ [69]. Allerdings wurden Patienten mit einer eGFR $<30 \mathrm{ml} / \mathrm{min} / 1,73 \mathrm{~m}^{2}$ oder mit einem Abfall der eGFR $>35 \%$ von der Studienteilnahme ausgeschlossen [68]. Bei 414 Patienten mit einer CKD im KDIGO GFR-Stadium G3b und einem Albumin/Kreatinin-Quotienten $>20 \mathrm{mg} / \mathrm{mmol}$ oder einer eGFR von 20-44 ml/min/1,73 $\mathrm{m}^{2}$ führte die Therapie mit Sacubitril/Valsartan im Vergleich zu Irbesartan zu einer vergleichbaren Reduktion der eGFR [70]. Künftig werden weitere Studien benötigt, die die Wirksamkeit und Sicherheit von ARNI bei Patienten mit CKD 4-5 untersuchen [68].

\section{Mineralokortikoid-Rezeptorantagonisten (MRA)}

Der Nutzen einer Angiotensin-Rezeptorblockade kann durch einen Renin/Angiotensinunabhängigen Anstieg des Serumaldosterons („Aldosteron-Escape“) limitiert sein [71]. Diesem soll durch den Einsatz von MRA entgegengewirkt werden [72]. Die steroidalen MRA Spironolacton und Eplerenon reduzierten in der RALES- und EMPHASIS-HF-Studie bei Patienten mit HFrEF die Sterblichkeit und die Anzahl der Hospitalisierungen (Tab. 6) [73, 74]. Die primären Endpunkte Gesamtsterblichkeit (RALES) sowie die Kombination aus kardiovaskulärer Sterblichkeit und Herzinsuffizienzhospitalisierung (EMPHASIS-HF) konnten bei Patienten mit einer eGFR von 30-60 ml/min/1,73 $\mathrm{m}^{2}$ im Vergleich zu Patienten mit einer eGFR $>60 \mathrm{ml} / \mathrm{min} / 1,73 \mathrm{~m}^{2}$ gleichermaßen reduziert werden [73, 74]. In der randomisierten DOHAS-Studie, wurden insgesamt 309 Hämodialyse-pflichtige Patienten entweder in die Therapiegruppe mit 25 mg Spironolacton pro Tag oder in die Kontrollgruppe randomisiert [75]. Im Vergleich zur Kontrollgruppe wurde der kombinierte primäre Endpunkt aus Mortalität und kardio- oder zerebrovaskulärer Hospitalisierung in der Spironolacton-Gruppe signifikant reduziert (HR 0,40; 95\%-Cl 0,20-0,81) [75]. Die kürzlich veröffentlichten Placebokontrollierten Spin-D (Safety and cardiovascular efficacy of spironolactone in dialysis dependent end-stage renal disease) und MiREnDa (Mineralocorticoid receptor antagonists in end-stage renal disease) Studien bestätigten die Sicherheit von Spironolacton unter einer engmaschigen Überwachung von Serumelektrolyten und Retentionsparameters bei Hämodialyse-pflichtigen Patienten [76, 77]. Die primären Wirksamkeitsendpunkte wurden nicht erreicht [76, 77]. Eine randomisierte, Placebo-kontrollierte Outcome-Studie (ALCHEMIST [NCT01848639]), die die Wirksamkeit von Spironolacton bei HämodialysePatienten mit Herzinsuffizienz untersucht, wird derzeit durchgeführt. Da bislang keine großen randomisierte Studien mit MRA bei Patienten mit HFrEF und fortgeschrittener, aber nicht Hämodialyse-pflichtiger, Niereninsuffizienz (KDIGO GFR-Stadium G4-5) durchgeführt wurden, sind Spironolacton und Eplerenon bei Patienten mit einer GFR $<30 \mathrm{ml} / \mathrm{min} / 1,73 \mathrm{~m}^{2}$ kontraindiziert (Abb. 3) [78]. Bei Hämodialyse-Patienten wurde die Sicherheit von Spironolacton unter engmaschigen laborchemischen Kontrollen gezeigt.

\section{Ivabradin}


Ivabradin hemmt den If-Kanal am Sinusknoten und kann entsprechend der Leitlinie bei Patienten mit einer LVEF $\leq 35 \%$, und einer Ruhe-Herzfrequenz $\geq 70$ Schlägen pro Minute im Sinusrhythmus trotz evidenzbasierter Therapie mit Betablockern, ACE-Hemmern (oder Angiotensin-Rezeptorblockern) und MRA gegeben werden. In der BEAUTIFUL-Studie bei Patienten mit dokumentierter KHK und HFrEF konnte im primären Endpunkt aus kardiovaskulärem Tod und Hospitalisierung wegen Herzinsuffizienz oder Myokardinfarkt kein signifikanter Unterschied zwischen Ivabradin und Placebo gezeigt werden (HR 1,00;95\%-Cl $0,91-1,10)[79]$. In der vordefinierten Subgruppe der Patienten mit einer Herzfrequenz $\geq 70$ Schlägen pro Minute konnte Ivabradin im Vergleich zu Placebo zwar nicht den primären Endpunkt (HR 0,91, 95\%-Cl 0,81-1,04) aber die Hospitalisierungsrate aufgrund tödlicher und nicht-tödlicher Myokardinfarkte reduzieren (HR 0,64, 95\%-Cl 0,49-0,84) [79]. Außerdem zeigte eine Subgruppen-Analyse der BEAUTIFUL-Studie, dass eine Herzfrequenz $\geq 70$ Schläge pro Minute im Vergleich zu einer niedrigen Herzfrequenz das Risiko eines kardiovaskulären Tods um 34\% und einer Herzinsuffizienzhospitalisierung um 53\% erhöhte [80]. In der randomisierten, doppelblinden SHIFT-Studie wurden 6.505 Patienten mit symptomatischer HFrEF und einer Ruheherzfrequenz $\geq 70$ Schlägen pro Minute untersucht [81]. Die Studie zeigte bei Patienten die mit Ivabradin behandelt wurden im Vergleich zu Placebo eine signifikante Reduktion des kombinierten primären Endpunkts aus kardiovaskulärer Sterblichkeit oder Herzinsuffizienzhospitalisierung (HR 0,82; 95\%-Cl 0,75-0,90) [81]. Die Inzidenz des primären Endpunkts konnte sowohl bei Patienten mit und ohne Nierenfunktionseinschränkung (eGFR $<60 \mathrm{~mL} / \mathrm{min} / 1,73 \mathrm{~m}^{2} ; \mathrm{HR} 0,82 ; \mathrm{p}=0,023$ vs. eGFR $\geq 60$ $\left.\mathrm{mL} / \mathrm{min} / 1,73 \mathrm{~m}^{2} ; \mathrm{HR} 0,81 ; \mathrm{p}<0,001\right)$ reduziert werden [82]. Da Ivabradin nur zu $20 \%$ renal ausgeschieden wird, ist bei einer eingeschränkten Nierenfunktion keine Dosisanpassungen erforderlich [51]. Allerdings wurde die Wirksamkeit von Ivabradin nicht gezielt bei Patienten mit einer fortgeschrittenen CKD untersucht (Tab. 7).

\section{Therapie einer Hyperkaliämie}

Die Unverträglichkeit ( $<50 \%$ der empfohlenen Dosis) einer Herzinsuffizienz-Therapie aus Angiotensin- oder Betablockade ist mit einem erhöhten Sterblichkeitsrisiko im Folgejahr assoziiert (ACE-Hemmer/Angiotensin-Rezeptor-Blocker: HR 1,72; 95\%-CI 1,43-2,01; Betablocker: HR 1,70; 95\%-CI 1,36-2,05) [83]. Häufige Ursachen für eine suboptimale Dosierung sind neben einer symptomatischen Hypotonie eine Verschlechterung der Nierenfunktion oder das Auftreten einer Hyperkaliämie [84]. Das Risiko einer Hyperkaliämie ist insbesondere bei Patienten mit einer fortgeschrittenen CKD oder einem Diabetes mellitus Typ II erhöht [85]. Patiromer ist ein nicht-absorbierbares, nicht-wasserlösliches Kationenaustauscher-Polymer, das vor allem im distalen Kolon überschüssiges Kalium bindet und somit die enterale Kaliumaufnahme reduziert [85]. In der Pearl-HF-Studie konnte Spironolacton unter Patiromer $(\mathrm{N}=55)$ im Vergleich zu Placebo $(\mathrm{N}=49)$ bei Patienten mit Herzinsuffizienz und hochnormalem Serumkalium (4,3-5,1 mmol/L) häufiger auf $50 \mathrm{mg} / \mathrm{d}$ aufdosiert werden ( $91 \%$ vs. $74 \%, p=0,019)$ [85]. Zudem traten Hyperkaliämien $>5,5 \mathrm{mmol} / \mathrm{I}$ unter Patiromer signifikant seltener auf (7,3\% vs. 24,5\%, $p=0,015)$ [85]. Von den 104 eingeschlossenen Patienten hatten etwa 60\% eine CKD [85]. Derzeit wird im Rahmen der CONTINUE-HF-Studie (EudraCT-Nummer 2017-003555-35) die Wirksamkeit von MRA mit Patiromer im Vergleich zur Standardtherapie zur Therapie einer HFrEF bei Patienten mit Hyperkaliämie untersucht. In der Placebo-kontrollierten DIAMOND-Endpunktstudie (NCT03888066) wird untersucht, ob bei Patienten mit HFrEF, die unter der Therapie mit RAAS-Inhibitoren eine Hyperkaliämie (Serumkalium $>5 \mathrm{mmol} / \mathrm{I}$ ) entwickeln, mit Hilfe von Patiromer eine leitliniengerechte Therapie etabliert und dadurch die Häufigkeit des 
kombinierten Endpunkts aus kardiovaskulärer Hospitalisierung und Sterblichkeit im Vergleich zu Placebo reduziert werden kann.

Neben Patiromer steht mit Natrium-Zirkonium-Cyclosilikat ein zweiter Kationenaustauscher zur oralen Behandlung einer Hyperkaliämie zur Verfügung, dessen kaliumsenkende Wirksamkeit und Sicherheit in der HARMONIZE-Studie gezeigt wurde [86].

\section{SGLT2-Inhibitoren}

SGLT2-Inhibitoren hemmen im proximalen Tubulus die Glukoserückresorption und erhöhen dadurch die renale Glukose-, Natrium- und Wasserausscheidung. Im Rahmen einer Placebokontrollierten Sicherheitsstudie für Empagliflozin zur Behandlung des Typ-2-Diabetes bei erhöhtem kardiovaskulären Risiko oder einer dokumentierten kardialen Erkrankung, wurde eine Abnahme des relativen Risikos für Herzinsuffizienzhospitalisierungen um 35\% verzeichnet (HR 0,65; 95\%-Cl 0,50-0,85) [87]. Interessanterweise konnten die Herzinsuffizienzendpunkte auch bei Patienten mit einer CKD (eGFR 30-60 ml/min/1,73 $\mathrm{m}^{2}$ ) reduziert werden, obwohl die blutzuckersenkende Wirkung mit zunehmender Nierenfunktionseinschränkung abnahm $[88,89]$. Auch im CANVAS-Studienprogramm mit Canagliflozin konnte das Risiko für herzinsuffizienzbezogene Endpunkte reduziert werden [90]. In der DECLARE-TIMI 58-Studie wurde die Wirkung von Dapagliflozin auf den koprimären Endpunkt kardiovaskulärer Tod und Herzinsuffizienzhospitalisierung untersucht. Dieser konnte durch die Therapie mit Dapagliflozin im Vergleich zu Placebo um 17\% reduziert werden (HR 0,83; 95\%-Cl 0,73-0,95) [91]. Eine Meta-Analyse der EMPAREGOUTCOME-, der CANVAS- und der DECLARE-TIMI 58-Studienprogramme zeigte bei Patienten mit einer schlechteren Nierenfunktion und somit höherem kardiovaskulären Risiko eine größere Reduktion des Risikos für Herzinsuffizienzhospitalisierungen und schwerwiegender kardiovaskulärer Ereignisse [92]. Eine Vielzahl von metabolischen und hämodynamischen Mechanismen werden als Ursache für die kardioprotektive Wirkung diskutiert. Neben der Reduktion der herzinsuffizienzbedingten Endpunkte bei Diabetes-Patienten gibt es Evidenz dafür, dass SGLT2-Inhibitoren das Fortschreiten einer CKD möglicherweise positiv beeinflussen können. Zwar fiel in der EMPA-REG OUTCOME-Studie unter Empagliflozin im Vergleich zu Placebo die eGFR während der ersten vier Wochen nach der Therapieinitiierung deutlicher ab (-0,77 vs. $0,01 \mathrm{ml} / \mathrm{min} / 1,73 \mathrm{~m}^{2} /$ Woche), blieb aber im weiteren Studienverlauf stabil und stieg nach Beendigung der SGLT2-Inhibiton wieder auf den Ausgangswert an [93]. In der Kontrollgruppe nahm die eGFR hingegen kontinuierlich ab [93]. Die kürzlich veröffentlichte CREDENCE-Studie konnte bei Patienten mit Diabetes und CKD KDIGO GFRStadium G2-4 (plus Albuminurie) die nephroprotektive Wirkung von Canagliflozin bestätigen [94]. Die Studie wurde nach der Durchführung einer prospektiv geplanten Interimsanalyse vorzeitig gestoppt, da Canagliflozin im Vergleich zu Placebo den kombinierten primären Endpunkt aus terminaler Niereninsuffizienz, Verdopplung des Serumkreatinins und kardiovaskulärem oder renalem Tod um 30\% reduzierte (HR 0,70; 95\%-Cl 0,59-0,82) [94]. Als Ursache für die nephroprotektive Wirkung wird der sogenannte tubuloglomeruläre Reflex diskutiert, bei dem es aufgrund einer erhöhten tubulären Natriumkonzentration im Bereich des juxtaglomerulären Apparats zu einer Vasokonstriktion der Vas afferens und dadurch zu einer Reduktion des glomerulären Filtrationsdrucks kommt [95]. Aktuell werden die DAPAHF- (NCT03036124) und die EMPEROR-HF-Studien (NCT03057977 für HFrEF und NCT03057951 für HFpEF) durchgeführt, die die Sicherheit und Wirksamkeit von Dapagliflozin beziehungsweise Empagliflozin bei Patienten mit Herzinsuffizienz mit und ohne Diabetes untersuchen. Da Patienten mit einer eGFR $<30 \mathrm{~mL} / \mathrm{min} / 1,73 \mathrm{~m}^{2}$ von den bisherigen Studien 
ausgeschlossen wurden (Tab. 8), können SGLT2-Inhibitoren bei terminaler CKD nicht empfohlen werden.

\section{Fazit für die Praxis}

- Niereninsuffiziente Patienten wurden aus Studien zur kardiovaskulären Pharmakotherapie oft ausgeschlossen und sind daher, gemessen an der Häufigkeit dieser Komorbidität, unterrepräsentiert. Da Therapieempfehlungen vor allem auf Beobachtungsstudien und Subgruppen-Analysen basieren, werden dringend prospektive randomisierte, kontrollierte Studien benötigt.

- Bei Patienten mit KHK ist die $\mathrm{PCl}$ ist im Vergleich zur Bypass-Operation mit einem erhöhten Risiko für Langzeitsterblichkeit, Myokardinfarkt und erneuter Revaskularisation assoziiert, wohingegen die kurzfristige Sterblichkeit und das Risiko zerebrovaskulärer Ereignisse nach $\mathrm{PCl}$ geringer ist.

- Bei herzinsuffizienten Patienten mit einer eGFR $\geq 30 \mathrm{~mL} / \mathrm{min} / 1,73 \mathrm{~m}^{2}$ unterscheidet sich die Herzinsuffizienztherapie im Wesentlichen nicht von der Therapie bei Nierengesunden.

- ACE-Hemmer/Angiotensin-Rezeptor-Blocker, Betablocker und Schleifendiuretika können unter Berücksichtigung der Nierenfunktion, Serumelektrolyte und Symptomatik bei fortgeschrittener Niereninsuffizienz (eGFR $<30 \mathrm{~mL} / \mathrm{min} / 1,73 \mathrm{~m}^{2}$ ) zur Therapie einer HFrEF eingesetzt werden. MRA sind bei einer eGFR $<30 \mathrm{~mL} / \mathrm{min} / 1,73$ $\mathrm{m}^{2}$ kontraindiziert.

- Weitere Studien werden benötigt, die die Wirksamkeit und Sicherheit von ARNI bei Patienten mit CKD 4-5 untersuchen.

\section{CME-Fragen}

1. Eine 67-jährige Patientin mit CKD (eGFR $20 \mathrm{ml} / \mathrm{min} / 1,73 \mathrm{~m}^{2}$ ) und HFrEF stellt sich aufgrund von zunehmender Dyspnoe und ausgeprägten peripheren Ödemen vor. Eine vor wenigen Tagen begonnene Therapie mit Torasemid hat bislang keine Besserung gebracht. Welche Aussage ist richtig?

A) Torasemid sollte zugunsten einer Monotherapie mit einem Thiaziddiuretikum abgesetzt werden.

B) Torasemid sollte durch Furosemid ersetzt werden, da Furosemid eine bessere orale Bioverfügbarkeit aufweist.

C) Die bisherige diuretische Therapie kann zur Etablierung einer sequentiellen Nephronblockade um ein Thiaziddiuretikum ergänzt werden.

D) In dieser Situation sind Schleifendiuretika streng kontraindiziert, da sie zu einer Verschlechterung der Nierenfunktion führen können.

E) Eine venovenöse Ultrafiltration sollte der medikamentösen Therapie aufgrund der geringeren Komplikationsrate vorgezogen werden.

2. Ein 55-jähriger Patient mit einer chronischen Nierenerkrankung im KDIGO GFR-Stadium G3 wird seit 6 Monaten mit Sacubitril/Valsartan und einem Betablocker behandelt. 
Bislang haben sich weder die Ejektionsfraktion (EF 28\%) noch die Belastungsdyspnoe NYHA III gebessert. Das Serumkalium war zuletzt auf $4,9 \mathrm{mmol} / \mathrm{L}$ angestiegen. Welche Aussage ist richtig?

A) Ein Mineralokortikoidrezeptorantagonist ist aufgrund der eingeschränkten Nierenfunktion kontraindiziert.

B) Patiromer sollte gegeben werden um das Kalium auf $<4 \mathrm{mmol} / \mathrm{L}$ zu senken.

C) Sacubitril/Valsartan sollte entsprechend der Leitlinien durch Spironolacton ersetzt werden.

D) Auf Schleifendiuretika sollte verzichtet werden, da diese das Risiko einer Hyperkaliämie erhöhen.

E) Die Herzinsuffizienztherapie sollte um einen Mineralokortikoidrezeptorantagonisten ergänzt werden.

3. Bei Patienten mit Hyperkaliämie gilt:

A) Bei einer ausgeprägten Hyperkaliämie $>6 \mathrm{mmol} / \mathrm{L}$ sollte Patiromer intravenös gegeben werden.

B) Patiromer ist das Mittel der Wahl zur Behandlung einer akuten Hyperkaliämie, da die Kaliumspiegel durch Patiromer sofort gesenkt werden können.

C) Patiromer ist bei gleichzeitiger Therapie mit einem Mineralokortikoidrezeptorantagonisten kontraindiziert.

D) Patiromer reduziert die enterale Kaliumaufnahme.

E) Natrium-Zirkonium-Cyclosilikat steigert als Kationenaustauscher die renale Kaliumexkretion.

4. Welche Aussage zu SGLT2-Inhibitoren ist richtig?

A) SGLT2-Inhibitoren steigern die tubuläre Glukoserückresorption.

B) Als Ursache für die nephroprotektiven Wirkung von SGLT2-Inhibitoren wird der sogenannte tubuloglomeruläre Reflex diskutiert.

C) Die blutzuckersenkende Wirkung nimmt mit zunehmender Nierenfunktionseinschränkung zu.

D) In der EMPA-REG OUTCOME-Studie kam es nach der Initiierung einer EmpagliflozinTherapie zu einem irreversiblen Abfall der eGFR.

E) SGLT2-Inhibitoren sind bei Albuminurie im KDIGO-Stadium A2 kontraindiziert.

5. Welche Aussage zur HFrEF trifft bei Patienten einer chronischen Nierenerkrankung zu:

A) Das Risiko eine Herzinsuffizienz zu entwickeln ist schon bei einer milden (eGFR 60-89 $\mathrm{ml} / \mathrm{min} / 1,73 \mathrm{~m}^{2}$ ) bis moderaten (eGFR 30-59 ml/min/1,73 $\mathrm{m}^{2}$ ) CKD erhöht.

B) In der PARADIGM-HF-Studie, reduzierte Valsartan/Sacubitril insbesondere bei Patienten mit einer chronischen Niereninsuffizienz in den KDIGO GFR-Stadien G4-5 die Gesamtsterblichkeit und die kardiovaskuläre Mortalität.

C) Betablocker sind bei Dialyse-Patienten immer kontraindiziert.

D) Im Rahmen des schwedischen Herzinsuffizienzregisters hatten Patienten mit fortgeschrittener Niereninsuffizienz unter RAS-Inhibition gegenüber Patienten ohne RAS-Inhibition eine deutlich erhöhte Sterblichkeit.

E) Steigt die Ejektionsfraktion unter einer adäquaten Herzinsuffizienztherapie $>40 \%$ sollte die Herzinsuffizienztherapie zugunsten der Nierenfunktion beendet werden. 
6. Welche Aussagen zur koronaren Herzkrankheit bei Patienten mit einer chronischen Nierenerkrankung trifft zu:
A) Die Komplikationen der KHK stellen die häufigsten Todesursachen von Patienten mit CKD dar.
B) FFR-Messungen sind bei Patienten mit einer Kreatinin-Clearance $\leq 45 \mathrm{ml} / \mathrm{min}$ häufig falsch-positiv (FFR $\leq 0,8)$.
C) Aufgrund der guten Sensitivität und Spezifität der nicht-invasiven Ischämiediagnostik sollte auf die Kontrastmittelexposition im Rahmen einer Koronarangiographie oder eines Kardio-CT verzichtet werden.
D) Das sekundäre kardiorenale Syndrom beschreibt das Auftreten einer chronischen Niereninsuffizienz infolge eines akuten Koronarsyndroms.
E) FFR-Messungen sollten bei Patienten mit chronischer Nierenerkrankung aufgrund des Kontrastmittelbedarfs vermieden werden.

7. Ein 70-jähriger niereninsuffizienter Patient (eGFR $35 \mathrm{ml} / \mathrm{min} / 1,73 \mathrm{~m}^{2}$ ) stellt sich bei zunehmender Angina pectoris elektiv zur Koronarrevaskularisation vor. Im Rahmen einer perkutanen Koronarintervention wird ein medikamentenbeschichteter Stent in den Ramus intermedius implantiert. Eine weitere Intervention ist zunächst nicht vorgesehen. Welche Aussage trifft zu?
A) Aufgrund des erhöhten
Blutungsrisikos
soll auf eine
Thrombozytenaggregationshemmung bei eingeschränkter Nierenfunktion verzichtet werden.
B) Eine duale Thrombozytenaggregationshemmung sollte zunächst initiiert werden. Fällt die eGFR unter $30 \mathrm{ml} / \mathrm{min} / 1,73 \mathrm{~m}^{2}$ sollte die antithrombotische Therapie pausiert werden.
C) Da Ticagrelor vor allem renal eliminiert wird, sollten Prasugrel und Clopidogrel zur Thrombozytenaggregationshemmung bevorzugt werden.
D) Eine duale Plättchenhemmung mit Aspirin und Clopidogrel für 6 Monate sollte initiiert werden.
E) Eine duale Plättchenhemmung sollte initiiert werden. Allerdings ist bei P2Y12-ADP- Rezeptorantagonisten eine Dosisanpassung erforderlich (zum Beispiel Prasugrel 5 $\mathrm{mg} / \mathrm{d})$.

8. Welche Aussage zur lipidsenkenden Therapie trifft bei niereninsuffizienten Patienten zu?
A) Bei Patienten mit fortgeschrittener Niereninsuffizienz sollten Fibrate aufgrund der besseren Evidenz gegenüber Statinen bevorzugt werden.
B) Die KDIGO-Leitlinie empfiehlt bei allen Patienten unter 50 Jahren mit fortgeschrittener Nierenfunktion eine lipidsenkende Therapie zu initiieren.
C) Die ESC/EAS-Leitlinien zur Diagnostik und Therapie von Dyslipidämien empfehlen bei allen Patienten mit nicht-dialysepflichtiger Niereninsuffizienz einen Ziel-LDL-Wert $<100 \mathrm{mg} / \mathrm{dL}$.
D) Für Kombinationen von Statinen und Ezetimib liegen bei Patienten mit eingeschränkter Nierenfunktion keine Daten vor, sodass Ezetimib in dieser Patientengruppe nicht eingesetzt werden sollte. 
E) Eine bestehende Statin-Therapie kann bei dialysepflichtigen Patienten fortgeführt werden.

9. Welche Aussage zur antianginösen Therapie trifft zu?

A) Da Angina pectoris bei niereninsuffizienten Patienten besonders häufig auftritt, hat die antianginöse Therapie in dieser Patientengruppe einen besonderen Stellenwert.

B) Nitrate können bei Patienten mit CKD eingesetzt werden, da diese vor allem hepatisch metabolisiert werden und daher weniger kumulieren.

C) Eine Kombinationstherapie aus Betablockern mit Verapamil sollte bei therapierefraktärer Angina pectoris erwogen werden.

D) Die medikamentöse Therapie der stabilen KHK dient ausschließlich der Symptomkontrolle.

E) Eine Kombinationstherapie mit Nitraten und Betablockern kann sinnvoll sein um eine reflektorische Tachykardie zu verhindern, die antianginöse Wirkung wird dadurch jedoch nicht verstärkt.

10. Welche Aussage zur Koronarrevaskularisation trifft bei niereninsuffizienten Patienten zu?

A) Bislang konnte keine Studie einen Vorteil einer Koronarrevaskularisation im Vergleich zu einer konservativen Therapie bei Patienten mit CKD zeigen.

B) Das Risiko für eine erneute Revaskularisation ist nach einer Bypass-Operation höher als nach einer perkutanen Koronarintervention.

C) Niereninsuffiziente Patienten haben nach einer Koronarrevaskularisation im Vergleich zu nierengesunden Patienten ein höheres Risiko für Re-Stenosen.

D) In der EXCEL-Studie war die Wahrscheinlichkeit eines akuten Nierenversagens nach perkutaner Koronarintervention höher als nach einer Bypass-Operation.

E) Auf eine perkutane Koronarintervention sollte aufgrund der damit einhergehenden Kontrastmittelexposition verzichtet werden.

Richtige Lösungen

$1 c, 2 e, 3 d, 4 b, 5 a, 6 a, 7 d, 8 e, 9 b, 10 c$

\section{Interessenkonflikt}

Dr. Lauder gibt an, dass kein Interessenskonflikt besteht. PD Dr. Ewen gibt an, Vortragshonorare von Pfizer, Novartis, Servier, AstraZeneca und Bayer erhalten zu haben. Dr. Emrich gibt an, dass sie Vortragshonorare von Pharmacosmos erhalten hat. Prof. Dr. Böhm erklärt, dass er Sprecherhonorare bzw. Honorare als wissenschaftlicher Berater von Amgen, Abbott, Astra-Zeneca, Bayer, Boehringer Ingelheim, Medtronic, Servier und Vifor erhalten hat. Prof. Dr. Mahfoud gibt an, Vortragshonorare von Medtronic, Recor, Berlin Chemie und Boehringer Ingelheim erhalten zu haben. Prof. Dr. Böhm und Prof. Dr. Mahfoud erklären, dass sie durch die Deutsche Forschungsgemeinschaft und den SFB-TRR218 unterstützt werden 


\section{Literatur}

1. Marx N, Noels H, Jankowski J et al (2018) Mechanisms of cardiovascular complications in chronic kidney disease: research focus of the Transregional Research Consortium SFB TRR219 of the University Hospital Aachen (RWTH) and the Saarland University. Clin Res Cardiol 107:120-126 . doi: 10.1007/s00392-018-1260-0

2. Tonelli M, Muntner P, Lloyd A et al (2012) Risk of coronary events in people with chronic kidney disease compared with those with diabetes: a population-level cohort study. Lancet 380:807-814 . doi: 10.1016/S0140-6736(12)60572-8

3. Kidney Disease: Improving Global Outcomes (KDIGO) CKD Work Group (2013) KDIGO 2012 Clinical Practice Guideline for the Evaluation and Management of Chronic Kidney Disease. Kidney Int Suppl 3:4-4 . doi: 10.1038/kisup.2012.76

4. Rangaswami J, Bhalla V, Blair JEA et al (2019) Cardiorenal syndrome: classification, pathophysiology, diagnosis, and treatment strategies: a scientific statement from the American Heart Association. Circulation 139:e840-e878 . doi: 10.1161/CIR.0000000000000664

5. Go AS, Chertow GM, Fan D et al (2004) Chronic kidney disease and the risks of death, cardiovascular events, and hospitalization. N Engl J Med 351:1296-1305 . doi: 10.1056/NEJMoa041031

6. Anavekar NS, McMurray JJV, Velazquez EJ et al (2004) Relation between renal dysfunction and cardiovascular outcomes after myocardial infarction. N Engl J Med 351:1285-1295 . doi: 10.1056/NEJMoa041365

7. Charytan D, Kuntz RE, Mauri L, DeFilippi C (2007) Distribution of coronary artery disease and relation to mortality in asymptomatic hemodialysis patients. Am J Kidney Dis 49:409-416 . doi: 10.1053/j.ajkd.2006.11.042

8. Schmidt A, Stefenelli T, Schuster E, Mayer G (2001) Informational Contribution of Noninvasive Screening Tests for Coronary Artery Disease in Patients on Chronic Renal Replacement Therapy. Am J Kidney Dis 37:56-63 . doi: 10.1053/ajkd.2001.20584

9. Bangalore S (2016) Diagnostic, therapeutic, and clinical trial conundrum of patients with chronic kidney disease. JACC Cardiovasc Interv 9:2110-2112 . doi: 10.1016/j.jcin.2016.08.031

10. Tebaldi M, Biscaglia S, Fineschi M et al (2016) Fractional flow reserve evaluation and chronic kidney disease: analysis from a multicenter Italian registry (the FREAK study). Catheter Cardiovasc Interv 88:555-562 . doi: 10.1002/ccd.26364

11. Mehran R, Dangas GD, Weisbord SD (2019) Contrast-associated acute kidney injury. N Engl J Med 380:2146-2155 . doi: 10.1056/NEJMra1805256

12. Ferrari R, Camici PG, Crea F et al (2018) A "diamond" approach to personalized treatment of angina. Nat Rev Cardiol 15:120-132 . doi: $10.1038 /$ nrcardio.2017.131

13. Montalescot G, Sechtem U, Achenbach S et al (2013) 2013 ESC guidelines on the management of stable coronary artery disease. Eur Heart J 34:2949-3003 . doi: 10.1093/eurheartj/eht296

14. Belsey J, Savelieva I, Mugelli A, Camm AJ (2015) Relative efficacy of antianginal drugs used as add-on therapy in patients with stable angina: A systematic review and meta-analysis. In: European Journal of Preventive Cardiology. pp 837-848

15. Jerling M (2006) Clinical pharmacokinetics of ranolazine. Clin Pharmacokinet 45:469-491 . doi: 10.2165/00003088-20064505000003

16. Borer JS, Fox K, Jaillon P, Lerebours G (2003) Antianginal and antiischemic effects of ivabradine, an If inhibitor, in atable angina. Circulation 107:817-823 . doi: 10.1161/01.CIR.0000048143.25023.87

17. Fox K, Ford I, Steg PG et al (2014) Ivabradine in stable coronary artery disease without clinical heart failure. N Engl J Med 371:1091-1099 . doi: 10.1056/NEJMoa1406430

18. Chaudhary R, Garg J, Krishnamoorthy P et al (2016) Ivabradine: heart failure and beyond. J Cardiovasc Pharmacol Ther 21:335343 . doi: $10.1177 / 1074248415624157$

19. Palmer SC, Di Micco L, Razavian M et al (2012) Effects of antiplatelet therapy on mortality and cardiovascular and bleeding outcomes in persons with chronic kidney disease. Ann Intern Med 156:445 . doi: 10.7326/0003-4819-156-6-201203200-00007

20. Gargiulo G, Santucci A, Piccolo R et al (2017) Impact of chronic kidney disease on 2-year clinical outcomes in patients treated with 6-month or 24-month DAPT duration: An analysis from the PRODIGY trial. Catheter Cardiovasc Interv 90:E73-E84 . doi: $10.1002 / \mathrm{ccd} .26921$

21. Schuett K, Savvaidis A, Maxeiner S et al (2017) Clot structure: A potent mortality risk factor in patients on hemodialysis. J Am Soc Nephrol 28:1622-1630 . doi: 10.1681/ASN.2016030336

22. Tanios BY, Itani HS, Zimmerman DL (2015) Clopidogrel use in end-stage kidney disease. Semin Dial 28:276-281 . doi: $10.1111 /$ sdi.12338

23. Baber U, Chandrasekhar J, Sartori S et al (2017) Associations between chronic kidney disease and outcomes with use of prasugrel versus clopidogrel in patients with acute coronary syndrome undergoing percutaneous coronary intervention. JACC CardiovasC Interv 10:2017-2025 . doi: 10.1016/j.jcin.2017.02.047

24. Bonello L, Angiolillo DJ, Aradi D, Sibbing D (2018) P2Y12 -ADP receptor blockade in chronic kidney disease patients with acute coronary syndromes. Circulation 138:1582-1596. doi: 10.1161/CIRCULATIONAHA.118.032078

25. Catapano AL, Graham I, De Backer G et al (2016) 2016 ESC/EAS Guidelines for the Management of Dyslipidaemias. Eur Heart J

26. Wanner C, Tonelli M (2014) KDIGO Clinical Practice Guideline for Lipid Management in CKD: summary of recommendation statements and clinical approach to the patient. Kidney Int 85:1303-1309 . doi: 10.1038/ki.2014.31

27. Baigent C, Landray MJ, Reith C et al (2011) The effects of lowering LDL cholesterol with simvastatin plus ezetimibe in patients with chronic kidney disease (Study of Heart and Renal Protection): a randomised placebo-controlled trial. Lancet 377:2181-2192 . doi: 10.1016/S0140-6736(11)60739-3

28. Tonelli M, Muntner P, Lloyd A et al (2014) Impact of age on the association between CKD and the risk of future coronary events Am J Kidney Dis 64:375-382 . doi: 10.1053/j.ajkd.2014.03.013 
29. Wanner C, Krane V, März W et al (2005) Atorvastatin in patients with type 2 diabetes mellitus undergoing hemodialysis. N Engl J Med 353:238-248 . doi: 10.1056/NEJMoa043545

30. Fellström BC, Jardine AG, Schmieder RE et al (2009) Rosuvastatin and cardiovascular events in patients undergoing hemodialysis. N Engl J Med 360:1395-1407 . doi: 10.1056/NEJMoa0810177

31. Chonchol M, Whittle J, Desbien A et al (2008) Chronic kidney disease is associated with angiographic coronary artery disease. Am J Nephrol 28:354-360 . doi: 10.1159/000111829

32. Attallah N, Yassine L, Fisher K, Yee J (2005) Risk of bleeding and restenosis among chronic kidney disease patients undergoing percutaneous coronary intervention. Clin Nephrol 64:412-418 . doi: 10.5414/CNP64412

33. Reddan DN, Szczech LA, Tuttle RH et al (2003) Chronic Kidney Disease, Mortality, and Treatment Strategies among Patients with Clinically Significant Coronary Artery Disease. J Am Soc Nephrol 14:2373-2380 . doi: 10.1097/01.ASN.0000083900.92829.F5

34. Milojevic M, Head SJ, Mack MJ et al (2018) The impact of chronic kidney disease on outcomes following percutaneous coronary intervention versus coronary artery bypass grafting in patients with complex coronary artery disease: five-year follow-up of the SYNTAX trial. Eurolntervention 14:102-111 . doi: 10.4244/EIJ-D-17-00620

35. Giustino G, Mehran R, Serruys PW et al (2018) Left main revascularization with PCl or CABG in patients with chronic kidney disease. J Am Coll Cardiol 72:754-765 . doi: 10.1016/j.jacc.2018.05.057

36. Wang Y, Zhu S, Gao P, Zhang Q (2017) Comparison of coronary artery bypass grafting and drug-eluting stents in patients with chronic kidney disease and multivessel disease: A meta-analysis. Eur J Intern Med 43:28-35 . doi: 10.1016/j.ejim.2017.04.002

37. Ponikowski P, Voors AA, Anker SD et al (2016) 2016 ESC Guidelines for the diagnosis and treatment of acute and chronic heart failure. Eur Heart J 37:2129-2200 . doi: 10.1093/eurheartj/ehw128

38. Kottgen A, Russell SD, Loehr LR et al (2007) Reduced kidney function as a risk factor for incident heart failure: the Atherosclerosis Risk in Communities (ARIC) Study. J Am Soc Nephrol 18:1307-1315 . doi: 10.1681/ASN.2006101159

39. Foley RN (2003) The clinical epidemiology of cardiovascular diseases in chronic kidney disease: clinical epidemiology of cardiac disease in dialysis patients: left ventricular hypertrophy, ischemic heart disease, and cardiac failure. Semin Dial 16:111-117 . doi: 10.1046/j.1525-139X.2003.160271.x

40. Erickson KF, Winkelmayer WC, Chertow GM, Bhattacharya J (2014) Physician visits and 30-day hospital readmissions in patients receiving hemodialysis. J Am Soc Nephrol 25:2079-2087 . doi: 10.1681/ASN.2013080879

41. Chen C, Yang X, Lei Y et al (2016) Urinary biomarkers at the time of AKI diagnosis as predictors of progression of AKI among patients with acute cardiorenal syndrome. Clin J Am Soc Nephrol 11:1536-1544 . doi: 10.2215/CJN.00910116

42. Jackson CE, MacDonald MR, Petrie MC et al (2011) Associations of albuminuria in patients with chronic heart failure: findings in the ALiskiren Observation of heart Failure Treatment study. Eur J Heart Fail 13:746-754 . doi: 10.1093/eurjhf/hfr031

43. Katz DH, Burns JA, Aguilar FG et al (2014) Albuminuria is independently associated with cardiac remodeling, abnormal right and left ventricular function, and worse outcomes in heart failure with preserved ejection fraction. JACC Hear Fail 2:586-596 . doi: 10.1016/j.jchf.2014.05.016

44. Velazquez EJ, Morrow DA, DeVore AD et al (2019) Angiotensin-neprilysin inhibition in acute decompensated heart failure. N Engl J Med 380:539-548 . doi: 10.1056/NEJMoa1812851

45. Seferovic PM, Ponikowski P, Anker SD et al (2019) Clinical practice update on heart failure 2019: pharmacotherapy, procedures, devices and patient management. An expert consensus meeting report of The Heart Failure Association of the European Society of Cardiology. Eur J Heart Fail ejhf.1531 . doi: 10.1002/ejhf.1531

46. Gheorghiade M, Vaduganathan M, Fonarow GC, Bonow RO (2013) Rehospitalization for heart failure. J Am Coll Cardiol 61:391403 . doi: 10.1016/j.jacc.2012.09.038

47. Ferreira JP, Santos M, Almeida S et al (2013) Tailoring diuretic therapy in acute heart failure: insight into early diuretic response predictors. Clin Res Cardiol 102:745-753 . doi: 10.1007/s00392-013-0588-8

48. Faris R, Flather M, Purcell H et al (2001) Diuretics for heart failure. In: Faris R (ed) Cochrane Database of Systematic Reviews. John Wiley \& Sons, Ltd, Chichester, UK

49. Faris R, Flather M, Purcell $\mathrm{H}$ et al (2002) Current evidence supporting the role of diuretics in heart failure: a meta analysis of randomised controlled trials. Int J Cardiol 82:149-158 . doi: 10.1016/S0167-5273(01)00600-3

50. Ellison DH, Felker GM (2017) Diuretic treatment in heart failure. N Engl J Med 377:1964-1975 . doi: 10.1056/NEJMra1703100

51. Damman K, Valente MAE, Voors AA et al (2014) Renal impairment, worsening renal function, and outcome in patients with heart failure: an updated meta-analysis. Eur Heart J 35:455-469 . doi: 10.1093/eurheartj/eht386

52. Testani JM, Chen J, McCauley BD et al (2010) Potential effects of aggressive decongestion during the treatment of decompensated heart failure on renal function and survival. Circulation 122:265-272 . doi: 10.1161/CIRCULATIONAHA.109.933275

53. Bart BA, Goldsmith SR, Lee KL et al (2012) Ultrafiltration in decompensated heart failure with cardiorenal syndrome. N Engl J Med 367:2296-2304 . doi: 10.1056/NEJMoa1210357

54. Frea S, Pidello S, Volpe A et al (2019) Diuretic treatment in high-risk acute decompensation of advanced chronic heart failurebolus intermittent vs. continuous infusion of furosemide: a randomized controlled trial. Clin Res Cardiol. doi: 10.1007/s00392-019-01521- $y$ 55. MERIT-HF Study Group (1999) Effect of metoprolol CR/XL in chronic heart failure: Metoprolol CR/XL Randomised Intervention Trial in-Congestive Heart Failure (MERIT-HF). Lancet 353:2001-2007 . doi: 10.1016/S0140-6736(99)04440-2

56. Dargie HJ, Lechat P (1999) The Cardiac Insufficiency Bisoprolol Study II (CIBIS-II): a randomised trial. Lancet 353:9-13 . doi: http://dx.doi.org/10.1016/S0140-6736(98)11181-9

57. Packer M, Bristow MR, Cohn JN et al (1996) The Effect of Carvedilol on Morbidity and Mortality in Patients with Chronic Heart Failure. N Engl J Med 334:1349-1355 . doi: 10.1056/NEJM199605233342101

58. Flather MD, Shibata MC, Coats AJS et al (2005) Randomized trial to determine the effect of nebivolol on mortality and cardiovascular hospital admission in elderly patients with heart failure (SENIORS). Eur Heart J 26:215-225 . doi: 10.1093/eurheartj/ehi115 
59. House AA, Wanner C, Sarnak MJ et al (2019) Heart failure in chronic kidney disease: conclusions from a Kidney Disease: Improving Global Outcomes (KDIGO) Controversies Conference. Kidney Int 95:1304-1317 . doi: 10.1016/j.kint.2019.02.022

60. Ghali JK, Wikstrand J, Van Veldhuisen DJ et al (2009) The influence of renal function on clinical outcome and response to $\beta$ blockade in systolic heart failure: insights from Metoprolol CR/XL Randomized Intervention Trial in Chronic HF (MERIT-HF). J Card Fail 15:310-318 . doi: 10.1016/j.cardfail.2008.11.003

61. Badve S V., Roberts MA, Hawley CM et al (2011) Effects of beta-adrenergic antagonists in patients with chronic kidney disease. J Am Coll Cardiol 58:1152-1161 . doi: 10.1016/j.jacc.2011.04.041

62. McDevitt DG (1987) Comparison of pharmacokinetic properties of beta-adrenoceptor blocking drugs. Eur Heart J 8:9-14 . doi: 10.1093/eurheartj/8.suppl_M.9

63. Cice G, Ferrara L, D’Andrea A et al (2003) Carvedilol increases two-year survival in dialysis patients with dilated cardiomyopathy. J Am Coll Cardiol 41:1438-1444 . doi: 10.1016/S0735-1097(03)00241-9

64. Weir MA, Dixon SN, Fleet JL et al (2015) B-blocker dialyzability and mortality in older patients receiving hemodialysis. J Am Soc Nephrol 26:987-996 . doi: 10.1681/ASN.2014040324

65. Bowling CB, Sanders PW, Allman RM et al (2013) Effects of enalapril in systolic heart failure patients with and without chronic kidney disease: Insights from the SOLVD Treatment trial. Int J Cardiol 167:151-156 . doi: 10.1016/j.ijcard.2011.12.056

66. Ljungman S, Kjekshus J, Swedberg K (1992) Renal function in severe congestive heart failure during treatment with enalapril (the Cooperative North Scandinavian Enalapril Survival Study [CONSENSUS] Trial). Am J Cardiol 70:479-487 . doi: 10.1016/0002-9149(92)911949

67. Edner M, Benson L, Dahlström U, Lund LH (2015) Association between renin-angiotensin system antagonist use and mortality in heart failure with severe renal insufficiency: a prospective propensity score-matched cohort study. Eur Heart J 36:2318-2326 . doi: 10.1093/eurheartj/ehv268

68. McMurray JJV, Packer M, Desai AS et al (2014) Angiotensin-neprilysin inhibition versus enalapril in heart failure. N Engl J Med 371:993-1004 . doi: 10.1056/NEJMoa1409077

69. Damman K, Gori M, Claggett B et al (2018) Renal effects and associated outcomes during angiotensin-neprilysin inhibition in heart failure. JACC Hear Fail 6:489-498 . doi: 10.1016/j.jchf.2018.02.004

70. Haynes R, Judge PK, Staplin N et al (2018) Effects of sacubitril/valsartan versus irbesartan in patients with chronic kidney disease. Circulation 138:1505-1514 . doi: 10.1161/CIRCULATIONAHA.118.034818

71. Athyros VG, Mikhailidis DP, Kakafika Al et al (2007) Angiotensin II reactivation and aldosterone escape phenomena in reninangiotensin-aldosterone system blockade: is oral renin inhibition the solution? Expert Opin Pharmacother 8:529-535 . doi: $10.1517 / 14656566.8 .5 .529$

72. van der Horst ICC, Voors AA, van Veldhuisen DJ (2007) Treatment of heart failure with ACE inhibitors and beta-blockers. Clin Res Cardiol 96:193-195 . doi: 10.1007/s00392-007-0487-y

73. Pitt B, Zannad F, Remme WJ et al (1999) The Effect of Spironolactone on Morbidity and Mortality in Patients with Severe Heart Failure. N Engl J Med 341:709-717 . doi: 10.1056/NEJM199909023411001

74. Zannad F, McMurray JJV, Krum H et al (2011) Eplerenone in patients with systolic heart failure and mild symptoms. N Engl J Med 364:11-21. doi: 10.1056/NEJMoa1009492

75. Matsumoto Y, Mori Y, Kageyama S et al (2014) Spironolactone reduces cardiovascular and cerebrovascular morbidity and mortality in hemodialysis patients. J Am Coll Cardiol 63:528-536 . doi: 10.1016/j.jacc.2013.09.056

76. Hammer F, Malzahn U, Donhauser J et al (2019) A randomized controlled trial of the effect of spironolactone on left ventricular mass in hemodialysis patients. Kidney Int 95:983-991 . doi: 10.1016/j.kint.2018.11.025

77. Charytan DM, Himmelfarb J, Ikizler TA et al (2019) Safety and cardiovascular efficacy of spironolactone in dialysis-dependent ESRD (SPin-D): a randomized, placebo-controlled, multiple dosage trial. Kidney Int 95:973-982 . doi: 10.1016/j.kint.2018.08.034

78. Pitt B, Pedro Ferreira J, Zannad F (2017) Mineralocorticoid receptor antagonists in patients with heart failure: current experience and future perspectives. Eur Hear J - Cardiovasc Pharmacother 3:48-57 . doi: 10.1093/ehjcvp/pvw016

79. Fox K, Ford I, Steg PG et al (2008) Ivabradine for patients with stable coronary artery disease and left-ventricular systolic dysfunction (BEAUTIFUL): a randomised, double-blind, placebo-controlled trial. Lancet 372:807-816 . doi: 10.1016/S0140-6736(08)61170-8

80. Fox K, Ford I, Steg PG et al (2008) Heart rate as a prognostic risk factor in patients with coronary artery disease and leftventricular systolic dysfunction (BEAUTIFUL): a subgroup analysis of a randomised controlled trial. Lancet 372:817-821 . doi: 10.1016/S0140-6736(08)61171-X

81. Swedberg K, Komajda M, Böhm M et al (2010) Ivabradine and outcomes in chronic heart failure (SHIFT): a randomised placebocontrolled study. Lancet 376:875-885 . doi: 10.1016/S0140-6736(10)61198-1

82. Voors AA, van Veldhuisen DJ, Robertson M et al (2014) The effect of heart rate reduction with ivabradine on renal function in patients with chronic heart failure: an analysis from SHIFT. Eur J Heart Fail 16:426-434 . doi: 10.1002/ejhf.59

83. Ouwerkerk W, Voors AA, Anker SD et al (2017) Determinants and clinical outcome of uptitration of ACE-inhibitors and betablockers in patients with heart failure: a prospective European study. Eur Heart J 38:1883-1890 . doi: 10.1093/eurheartj/ehx026

84. Maggioni AP, Anker SD, Dahlström U et al (2013) Are hospitalized or ambulatory patients with heart failure treated in accordance with European Society of Cardiology guidelines? Evidence from 12440 patients of the ESC Heart Failure Long-Term Registry. Eur J Heart Fail 15:1173-1184 . doi: 10.1093/eurjhf/hft134

85. Pitt B, Anker SD, Bushinsky DA et al (2011) Evaluation of the efficacy and safety of RLY5016, a polymeric potassium binder, in a double-blind, placebo-controlled study in patients with chronic heart failure (the PEARL-HF) trial. Eur Heart J 32:820-828 . doi: 10.1093/eurheartj/ehq502

86. Anker SD, Kosiborod M, Zannad F et al (2015) Maintenance of serum potassium with sodium zirconium cyclosilicate (ZS-9) in heart failure patients: results from a phase 3 randomized, double-blind, placebo-controlled trial. Eur J Heart Fail 17:1050-1056 . doi: 10.1002/ejhf.300 
87. Zinman B, Wanner C, Lachin JM et al (2015) Empagliflozin, cardiovascular outcomes, and mortality in type 2 diabetes. N Engl J Med 373:2117-2128 . doi: 10.1056/NEJMoa1504720

88. Wanner C, Lachin JM, Inzucchi SE et al (2018) Empagliflozin and clinical outcomes in patients with type 2 diabetes mellitus, established cardiovascular disease, and chronic kidney cisease. Circulation 137:119-129 . doi: 10.1161/CIRCULATIONAHA.117.028268

89. Inzucchi SE, Kosiborod M, Fitchett D et al (2018) Improvement in cardiovascular outcomes with empagliflozin Is independent of glycemic control. Circulation 138:1904-1907 . doi: 10.1161/CIRCULATIONAHA.118.035759

90. Rådholm K, Figtree G, Perkovic V et al (2018) Canagliflozin and heart failure in type 2 diabetes mellitus. Circulation 138:458-468 . doi: 10.1161/CIRCULATIONAHA.118.034222

91. Wiviott SD, Raz I, Bonaca MP et al (2019) Dapagliflozin and cardiovascular outcomes in type 2 diabetes. N Engl J Med 380:347357 . doi: 10.1056/NEJMoa1812389

92. Zelniker TA, Wiviott SD, Raz I et al (2019) SGLT2 inhibitors for primary and secondary prevention of cardiovascular and renal outcomes in type 2 diabetes: a systematic review and meta-analysis of cardiovascular outcome trials. Lancet 393:31-39 . doi: 10.1016/S0140-6736(18)32590-X

93. Wanner C, Heerspink HJL, Zinman B et al (2018) Empagliflozin and kidney function decline in patients with type 2 diabetes: a slope analysis from the EMPA-REG OUTCOME Trial. J Am Soc Nephrol 29:ASN.2018010103 . doi: 10.1681/ASN.2018010103

94. Perkovic V, Jardine MJ, Neal B et al (2019) Canagliflozin and renal outcomes in type 2 diabetes and nephropathy. N Engl J Med 380:2295-2306 . doi: 10.1056/NEJMoa1811744

95. Ghosh RK, Bandyopadhyay D, Hajra A et al (2016) Cardiovascular outcomes of sodium-glucose cotransporter 2 inhibitors: A comprehensive review of clinical and preclinical studies. Int J Cardiol 212:29-36 . doi: 10.1016/j.ijcard.2016.02.134

\section{Abbildungslegenden}

Abbildung 1. Pathophysiologische Mechanismen der Interaktion zwischen Herz und Niere. FGF-23 = Fibroblasten-Wachstumsfaktor 23; HZV = Herzzeitvolumen; PTH = Parathormon; SNS = Sympathisches Nervensystem; Vit. $D=$ Vitamin D.

Abbildung 2. Stadieneinteilung der chronischen Niereninsuffizienz nach KDIGO [3]. Grün: niedriges Risiko; gelb: moderat erhöhtes Risiko; orange: hohes Risiko; rot: sehr hohes Risiko.

Abbildung 3. Therapiealgorithmus bei Patienten mit HFrEF und fortgeschrittener Niereninsuffizienz (modifiziert nach $[37,45]$ )

\section{Tabellen}

\begin{tabular}{|l|l|l|}
\hline \multicolumn{3}{|l|}{ Tabelle 1. Klassifikation des kardiorenalen Syndroms } \\
\hline Typ & Bezeichnung & Beispiel \\
\hline 1 & Akutes kardiorenales Syndrom & $\begin{array}{l}\text { Akute Herzinsuffizienz, akutes } \\
\text { Koronarsyndrom oder kardiogener Schock } \\
\rightarrow \text { akutes Nierenversagen }\end{array}$ \\
\hline 2 & Chronisches kardiorenales Syndrom & $\begin{array}{l}\text { Chronische Herzinsuffizienz } \rightarrow \text { chronische } \\
\text { Niereninsuffizienz }\end{array}$ \\
\hline 3 & Akutes renokardiales Syndrom & $\begin{array}{l}\text { Akutes Nierenversagen } \rightarrow \text { akute } \\
\text { Herzinsuffizienz, Arrhythmien oder akutes } \\
\text { Koronarsyndrom }\end{array}$ \\
\hline 4 & Chronisches renokardiales Syndrom & Chronische Niereninsuffizienz $\rightarrow$ chronische \\
\hline
\end{tabular}




\begin{tabular}{|l|l|l|}
\hline & & Herzinsuffizienz \\
\hline 5 & Sekundäres kardiorenales Syndrom & $\begin{array}{l}\text { Systemische Erkrankung (z.B. Sepsis oder } \\
\text { Amyloidose) } \rightarrow \text { Herz- und } \\
\text { Niereninsuffizienz }\end{array}$ \\
\hline
\end{tabular}

Tabelle 2. Thrombozytenaggregationshemmung bei chronischer Niereninsuffizienz (CKD) (modifiziert nach Bonello et al. [24])

\begin{tabular}{|l|l|l|l|l|}
\hline & $\begin{array}{l}\text { Acetylsalicylsäure } \\
\text { (ASS) }\end{array}$ & Clopidogrel & Prasugrel & Ticagrelor \\
\hline $\begin{array}{l}\text { Wirk- } \\
\text { mechanismus }\end{array}$ & $\begin{array}{l}\text { V.a. Hemmung } \\
\text { von COX-1 }\end{array}$ & \multicolumn{2}{|l|}{ P2Y12-ADP-Rezeptorantagonist } \\
\hline Reversibilität & Nein & Nein & Nein & Ja \\
\hline Prodrug & Nein & Ja & Ja & Nein \\
\hline Metabolisierung & Hepatisch & $\begin{array}{l}\text { Hepatisch (CYP- } \\
\text { abhängig) }\end{array}$ & $\begin{array}{l}\text { Hepatisch (CYP- } \\
\text { abhängig) }\end{array}$ & $\begin{array}{l}\text { Hepatisch (CYP- } \\
\text { abhängig) }\end{array}$ \\
\hline Elimination & Renal (80-100\%) & $\begin{array}{l}\text { Renal (50\%), } \\
\text { fäkal (50\%) }\end{array}$ & $\begin{array}{l}\text { Renal (68\%), } \\
\text { fäkal (27\%) }\end{array}$ & $\begin{array}{l}\text { Renal <1\%, } \\
\text { fäkal }\end{array}$ \\
\hline $\begin{array}{l}\text { Einfluss auf } \\
\text { Nierenfunktion }\end{array}$ & Nein & Nein & Nein & $\begin{array}{l}\text { Kreatinin- } \\
\text { anstieg möglich }\end{array}$ \\
\hline $\begin{array}{l}\text { Dosisreduktion } \\
\text { bei CKD nötig? }\end{array}$ & Nein & Nein & Nein & Nein \\
\hline
\end{tabular}

\begin{tabular}{|c|c|c|c|c|c|c|}
\hline Studie & Jahr & $\begin{array}{l}\text { Wirkstoff } \\
\text { (vs. } \\
\text { Placebo) }\end{array}$ & $\mathbf{N}$ & $\begin{array}{l}\text { CKD } \\
* \\
(\%)\end{array}$ & $\begin{array}{l}\text { Einschlusskriteri } \\
\text { um }\end{array}$ & $\begin{array}{l}\text { Renale } \\
\text { Ausschlusskriterien }\end{array}$ \\
\hline MDC & 1993 & $\begin{array}{l}\text { Metoprol } \\
\text { ol }\end{array}$ & 383 & $\mathrm{~N} / \mathrm{A}$ & $\begin{array}{l}\text { LVEF <40\%; Alter } \\
\text { 16-75 Jahre }\end{array}$ & \\
\hline CIBIS & 1994 & Bisoprolol & 641 & $\mathrm{~N} / \mathrm{A}$ & $\begin{array}{l}\text { LVEF <40\%; Alter } \\
\text { 18-75 Jahre; } \\
\text { NYHA III/IV }\end{array}$ & $\begin{array}{l}\text { Kreatinin }>3,4 \mathrm{mg} / \mathrm{dL}(>300 \\
\mu \mathrm{mol} / \mathrm{L})\end{array}$ \\
\hline $\begin{array}{l}\text { US- } \\
\text { Carvedilol }\end{array}$ & 1996 & Carvedilol & $\begin{array}{l}109 \\
4\end{array}$ & $\mathrm{~N} / \mathrm{A}$ & LVEF $\leq 35 \%$ & $\begin{array}{l}\text { Klinisch relevante } \\
\text { Nierenerkrankung }\end{array}$ \\
\hline ANZ & 1997 & Carvedilol & 415 & $\mathrm{~N} / \mathrm{A}$ & $\begin{array}{l}\text { LVEF <45\%; } \\
\text { ischämische } \\
\text { Kardiomyopathie } \\
\text {; NYHA II/III }\end{array}$ & $\begin{array}{l}\text { Kreatinin >2,83 mg/dL } \\
\text { (>250 } \mu \mathrm{mol} / \mathrm{L})\end{array}$ \\
\hline CIBIS II & 1999 & Bisoprolol & $\begin{array}{l}264 \\
7\end{array}$ & 43 & $\begin{array}{l}\text { LVEF <35\%; Alter } \\
\text { 18-80 Jahre; } \\
\text { NYHA III/IV }\end{array}$ & $\begin{array}{l}\text { Kreatinin }>3,4 \mathrm{mg} / \mathrm{dL}(>300 \\
\mu \mathrm{mol} / \mathrm{L})\end{array}$ \\
\hline MERIT-HF & 1999 & Metoprol & 399 & 37 & LVEF $\leq 40 \%$; Alter & \\
\hline
\end{tabular}




\begin{tabular}{|c|c|c|c|c|c|c|}
\hline & & ol & 1 & & $\begin{array}{l}\text { 40-80 Jahre; } \\
\text { NHYA III/IV }\end{array}$ & \\
\hline $\begin{array}{l}\text { COPERNIC } \\
\text { US }\end{array}$ & 2001 & Carvedilol & $\begin{array}{l}228 \\
9\end{array}$ & \multirow[t]{2}{*}{$61^{+}$} & $\begin{array}{l}\text { LVEF <25\%; } \\
\text { NYHA III/IV }\end{array}$ & $\begin{array}{l}\text { Kreatinin }>2,8 \mathrm{mg} / \mathrm{dL} \\
\text { (>247,5 } \mu \mathrm{mol} / \mathrm{L})\end{array}$ \\
\hline $\begin{array}{l}\text { CAPRICOR } \\
\mathrm{N}\end{array}$ & 2001 & Carvedilol & $\begin{array}{l}195 \\
9\end{array}$ & & $\begin{array}{l}\text { 3-21 Tage nach } \\
\text { einem } \\
\text { Myokardinfarkt, } \\
\text { LVEF } \leq 40 \%\end{array}$ & $\begin{array}{l}\text { Nierenfunktionseinschränk } \\
\text { ung }\end{array}$ \\
\hline BEST & 2001 & Bucindolo & $\begin{array}{l}270 \\
8\end{array}$ & $\mathrm{~N} / \mathrm{A}$ & $\begin{array}{l}\text { LVEF <35\%; } \\
\text { NYHA III/IV }\end{array}$ & $\begin{array}{l}\text { Kreatinin }>3,0 \mathrm{mg} / \mathrm{dL}(>265 \\
\mu \mathrm{mol} / \mathrm{L})\end{array}$ \\
\hline $\begin{array}{l}\text { CHRISTM } \\
\text { AS }\end{array}$ & 2003 & Carvedilol & 387 & $\mathrm{~N} / \mathrm{A}$ & $\begin{array}{l}\text { LVEF }<40 \% \text {; } \\
\text { ischämische } \\
\text { Kardiomyopathie } \\
\text {; Alter } \geq 40 \text { Jahre; } \\
\text { NYHA I-III }\end{array}$ & $\begin{array}{l}\text { Klinisch relevante } \\
\text { Nierenerkrankung }\end{array}$ \\
\hline SENIORS & 2005 & Nebivolol & $\begin{array}{l}212 \\
8\end{array}$ & 42 & $\begin{array}{l}\text { LVEF } \leq 35 \% ; \\
\text { Herzinsuffizienz- } \\
\text { Hospitalisierung } \\
\text { innerhalb der } \\
\text { letzten } 12 \\
\text { Monate, Alter } \\
\geq 70 \text { Jahre }\end{array}$ & $\begin{array}{l}\text { Signifikante } \\
\text { Nierenerkrankung }\end{array}$ \\
\hline
\end{tabular}

Tabelle 4. Charakteristika wichtiger Studien mit ACE-Hemmern

\begin{tabular}{|c|c|c|c|c|c|c|c|}
\hline Studie & Jahr & $\begin{array}{l}\text { Wirkstoff } \\
\text { (vs. } \\
\text { Placebo) }\end{array}$ & $\mathbf{N}$ & $\begin{array}{l}\text { CKD* } \\
\text { (\%) }\end{array}$ & Einschlusskriterium & \multicolumn{2}{|c|}{$\begin{array}{l}\text { Renale } \\
\text { Ausschlusskriterien }\end{array}$} \\
\hline CONSENSUS & 1987 & Enalapril & 253 & $\mathrm{~N} / \mathrm{A}$ & NYHA IV & $\begin{array}{l}\text { Kreatinin } \\
\mathrm{mg} / \mathrm{dL} \\
\mu \mathrm{mol} / \mathrm{L})\end{array}$ & $\begin{array}{r}>3,4 \\
(>300\end{array}$ \\
\hline $\begin{array}{l}\text { SOLVD- } \\
\text { Treatment }\end{array}$ & 1991 & Enalapril & 2569 & 36 & $\begin{array}{l}\text { LVEF } \leq 35 \% ; \quad \text { Alter } \\
21-80 \quad \text { Jahre; } \\
\text { symptomatisch } \\
\text { nach Run-in }\end{array}$ & $\begin{array}{l}\text { Kreatinin } \\
\mathrm{mg} / \mathrm{dL} \\
\mu \mathrm{mol} / \mathrm{L})\end{array}$ & $\begin{array}{r}>2,0 \\
(>177\end{array}$ \\
\hline $\begin{array}{l}\text { SOLVD- } \\
\text { Prevention }\end{array}$ & 1991 & Enalapril & 4228 & 21 & $\begin{array}{l}\text { LVEF } \leq 35 \% ; \text { Alter } \\
21-80 \quad \text { Jahre, } \\
\text { asymptomatisch } \\
\text { nach Run-in }\end{array}$ & $\begin{array}{l}\text { Kreatinin } \\
\mathrm{mg} / \mathrm{dL} \\
\mu \mathrm{mol} / \mathrm{L})\end{array}$ & $\begin{array}{r}>2,0 \\
(>177\end{array}$ \\
\hline SAVE & 1992 & Captopril & 2321 & 33 & $\begin{array}{l}\text { LVEF } \leq 40 \% \text { nach } \\
\text { Myokardinfarkt; } \\
\text { Alter } 21-80 \text { Jahre }\end{array}$ & $\begin{array}{l}\text { Kreatinin } \\
\mathrm{mg} / \mathrm{dL} \\
\mu \mathrm{mol} / \mathrm{L})\end{array}$ & $\begin{array}{r}>2,5 \\
(>221\end{array}$ \\
\hline
\end{tabular}




\begin{tabular}{|c|c|c|c|c|c|c|c|}
\hline AIRE & 1993 & Ramipril & 2006 & N/A & $\begin{array}{l}\text { Herzinsuffizienz } \\
\text { nach akutem } \\
\text { Myokardinfarkt, } \\
\text { Alter } \geq 18 \text { Jahre, } \\
\text { NYHA I-III }\end{array}$ & $\mathrm{N} / \mathrm{A}$ & \\
\hline TRACE & 1995 & Trandolapril & 1749 & 40 & $\begin{array}{l}\text { Linksventrikuläre } \\
\text { Dysfunktion nach } \\
\text { Myokardinfarkt; } \\
\text { Alter } \geq 18 \text { Jahre }\end{array}$ & $\begin{array}{l}\text { Kreatinin } \\
\mathrm{mg} / \mathrm{dL} \\
\mu \mathrm{mol} / \mathrm{L})\end{array}$ & $\begin{array}{r}>2,3 \\
(>200\end{array}$ \\
\hline
\end{tabular}

\begin{tabular}{|c|c|c|c|c|c|c|}
\hline Studie & Jahr & $\begin{array}{l}\text { Wirkstoff } \\
\text { (vs. } \\
\text { Placebo) }\end{array}$ & $\mathbf{N}$ & $\begin{array}{l}\text { CKD* } \\
\text { (\%) }\end{array}$ & Einschlusskriterium & $\begin{array}{l}\text { Renale } \\
\text { Ausschlusskriterien }\end{array}$ \\
\hline Val-HeFT & 2001 & Valsartan & 5010 & 58 & $\begin{array}{l}\text { LVEF } \leq 40 \% \text {; Alter } \\
\geq 18 \text { Jahre; NYHA II- } \\
\text { IV }\end{array}$ & $\begin{array}{l}\text { Kreatinin }>2,5 \\
\mathrm{mg} / \mathrm{dL}(>221 \\
\mu \mathrm{mol} / \mathrm{L})\end{array}$ \\
\hline $\begin{array}{l}\text { CHARM- } \\
\text { Added }\end{array}$ & 2003 & Candesartan & 2548 & 33 & $\begin{array}{l}\text { LVEF } \leq 40 \% \text {; Alter } \\
\geq 18 \text { Jahre; NYHA II- } \\
\text { IV }\end{array}$ & $\begin{array}{l}\text { Kreatinin }>3,0 \\
\mathrm{mg} / \mathrm{dL}(>265 \\
\mu \mathrm{mol} / \mathrm{L})\end{array}$ \\
\hline $\begin{array}{l}\text { CHARM- } \\
\text { Alternative }\end{array}$ & 2003 & Candesartan & 2028 & 43 & $\begin{array}{l}\text { LVEF } \leq 40 \% \text {; Alter } \\
\geq 18 \text { Jahre; NYHA II- } \\
\text { IV }\end{array}$ & $\begin{array}{l}\text { Kreatinin }>3,0 \\
\mathrm{mg} / \mathrm{dL}(>265 \\
\mu \mathrm{mol} / \mathrm{L})\end{array}$ \\
\hline
\end{tabular}

\begin{tabular}{|l|l|l|l|l|l|l|}
\hline \multicolumn{2}{|l|}{ Tabelle 6. Charakteristika wichtiger Studien mit Mineralokortikoid-Rezeptorantagonisten } \\
\hline Studie & Jahr & $\begin{array}{l}\text { Wirkstoff (vs. } \\
\text { Placebo) }\end{array}$ & $\mathbf{N}$ & $\begin{array}{l}\text { CKD } \\
*(\%)\end{array}$ & $\begin{array}{l}\text { Einschlusskriteriu } \\
\mathbf{m}\end{array}$ & $\begin{array}{l}\text { Renale } \\
\text { Ausschlusskriterie } \\
\mathbf{n}\end{array}$ \\
\hline RALES & $\begin{array}{l}199 \\
9\end{array}$ & $\begin{array}{l}\text { Spironolacto } \\
\mathrm{n}\end{array}$ & $\begin{array}{l}1.66 \\
3\end{array}$ & 48 & $\begin{array}{l}\text { LVEF } \leq 35 \% ; \text { NYHA } \\
\text { III/IV }\end{array}$ & $\begin{array}{l}\text { Kreatinin }>2,5 \\
\mathrm{mg} / \mathrm{dL}(>221 \\
\mu \mathrm{mol} / \mathrm{L})\end{array}$ \\
\hline EPHESUS & $\begin{array}{l}200 \\
3\end{array}$ & Eplerenon & $\begin{array}{l}6.63 \\
2\end{array}$ & 33 & $\begin{array}{l}\text { LVEF } \leq 40 \% \text { nach } \\
\text { Myokardinfarkt }\end{array}$ & $\begin{array}{l}\text { Kreatinin }>2,5 \\
\mathrm{mg} / \mathrm{dL}(>221 \\
\mu \mathrm{mol} / \mathrm{L})\end{array}$ \\
\hline $\begin{array}{l}\text { EMPHASIS } \\
\text {-HF }\end{array}$ & $\begin{array}{l}201 \\
1\end{array}$ & Eplerenon & $\begin{array}{l}2.73 \\
7\end{array}$ & 33 & $\begin{array}{l}\text { LVEF } \leq 35 \% ; \text { Alter } \\
\geq 55 \text { Jahre; NYHA II }\end{array}$ & $\begin{array}{l}\text { eGFR }<30 \\
\mathrm{ml} / \mathrm{min} / 1,73 \mathrm{~m}^{2}\end{array}$ \\
\hline *GFR <60 ml/min/1,73 $\mathrm{m}^{2}$. LVEF $=$ Linksventrikuläre Ejektionsfraktion. \\
\hline
\end{tabular}




\begin{tabular}{|l|l|l|l|l|l|l|}
\hline \multicolumn{2}{|l|}{ Tabelle 7. Charakteristika wichtiger Studien mit Ivabradin } \\
\hline Studie & Jahr & $\begin{array}{l}\text { Wirkstoff } \\
\text { (vs. } \\
\text { Placebo) }\end{array}$ & $\mathbf{N}$ & $\begin{array}{l}\text { CKD* } \\
\text { (\%) }\end{array}$ & Einschlusskriterium & $\begin{array}{l}\text { Renale } \\
\text { Ausschlusskriterien }\end{array}$ \\
\hline BEAUTIFUL & 2008 & Ivabradin & 10.917 & 48 & $\begin{array}{l}\text { KHK; LVEF <40\%; } \\
\text { Sinusrhythmus mit } \\
\text { Ruhe-HF HF } \\
\geq 60 / \text { min; Alter } \geq 55 \\
\text { Jahre (Diabetiker } \\
\geq 18 \text { Jahre) }\end{array}$ & N/A \\
\hline SHIFT & 2010 & Ivabradin & 6.558 & $24 \%$ & $\begin{array}{l}\text { LVEF } \leq 35 \% ; \\
\text { Sinusrhythmus mit } \\
\text { Ruhe-HF } \geq 70 / m i n ; \\
\text { Alter } \geq 18 \text { Jahre }\end{array}$ & $\begin{array}{l}\text { Kreatinin }>2,5 \\
\text { mg/dL (>220 } \\
\mu \text { mol/L) }\end{array}$ \\
\hline
\end{tabular}

\begin{tabular}{|c|c|c|c|c|c|c|}
\hline Studie & Jahr & $\begin{array}{l}\text { Wirkstoff } \\
\text { (vs. } \\
\text { Placebo) }\end{array}$ & $\mathbf{N}$ & $\begin{array}{l}\text { CKD } \\
*(\%)\end{array}$ & $\begin{array}{l}\text { Einschlusskriteriu } \\
\mathrm{m}\end{array}$ & $\begin{array}{l}\text { Renale } \\
\text { Ausschlusskriterie } \\
\mathrm{n}\end{array}$ \\
\hline $\begin{array}{l}\text { EMPA- } \\
\text { REG } \\
\text { OUTCOM } \\
\text { E }\end{array}$ & $\begin{array}{l}201 \\
5\end{array}$ & $\begin{array}{l}\text { Empagliflozi } \\
n\end{array}$ & 7.020 & 26 & $\begin{array}{l}\text { Typ } 2 \text { Diabetes + } \\
\text { hohes } \\
\text { kardiovaskuläres } \\
\text { Risiko }\end{array}$ & $\begin{array}{l}\text { eGFR }<30 \\
\mathrm{ml} / \mathrm{min} / 1,73 \mathrm{~m}^{2}\end{array}$ \\
\hline CANVAS & $\begin{array}{l}201 \\
8\end{array}$ & Canagliflozin & 10.143 & $\mathrm{~N} / \mathrm{A}$ & $\begin{array}{l}\text { Typ } 2 \text { Diabetes + } \\
\text { hohes } \\
\text { kardiovaskuläres } \\
\text { Risiko }\end{array}$ & $\begin{array}{l}\mathrm{eGFR}<30 \\
\mathrm{ml} / \mathrm{min} / 1,73 \mathrm{~m}^{2}\end{array}$ \\
\hline $\begin{array}{l}\text { DECLARE- } \\
\text { TIMI }\end{array}$ & $\begin{array}{l}201 \\
9\end{array}$ & $\begin{array}{l}\text { Dapagliflozi } \\
n\end{array}$ & 17.160 & $7 \%$ & $\begin{array}{l}\text { Typ } 2 \text { Diabetes + } \\
\text { hohes } \\
\text { kardiovaskuläres } \\
\text { Risiko oder } \\
\text { kardiovaskuläre } \\
\text { Erkrankung; Alter } \\
\geq 40 \text { Jahre }\end{array}$ & $\begin{array}{l}\text { Krea-Clearance } \\
<60 \mathrm{ml} / \mathrm{min}\end{array}$ \\
\hline $\begin{array}{l}\text { CREDENC } \\
\text { E }\end{array}$ & $\begin{array}{l}201 \\
9\end{array}$ & Canagliflozin & 4401 & $60 \%$ & $\begin{array}{l}\text { Typ } 2 \text { Diabetes } \\
\text { und CKD mit } \\
\text { Albuminurie } \\
\text { (UACR >300 und } \\
\leq 5.000 \mathrm{mg} / \mathrm{g} \text {; Alter } \\
\geq 30 \text { Jahre }\end{array}$ & $\begin{array}{l}\text { eGFR }<30 \\
\mathrm{ml} / \mathrm{min} / 1,73 \mathrm{~m}^{2}\end{array}$ \\
\hline DAPA-HF & $\mathrm{N} / \mathrm{A}$ & $\begin{array}{l}\text { Dapagliflozi } \\
n\end{array}$ & $\begin{array}{l}4744 \\
\text { (geplant } \\
\text { ) }\end{array}$ & $\mathrm{N} / \mathrm{A}$ & $\begin{array}{l}\text { NYHA II-IV; LVEF } \\
\leq 40 \% \text {; Alter } \geq 18 \\
\text { Jahre }\end{array}$ & $\begin{array}{l}\mathrm{eGFR}<30 \\
\mathrm{ml} / \mathrm{min} / 1,73 \mathrm{~m}^{2}\end{array}$ \\
\hline
\end{tabular}




\begin{tabular}{|c|c|c|c|c|c|c|}
\hline $\begin{array}{l}\text { EMPEROR } \\
\text { - } \\
\text { Preserved }\end{array}$ & $\mathrm{N} / \mathrm{A}$ & $\begin{array}{l}\text { Empagliflozi } \\
\mathrm{n}\end{array}$ & $\begin{array}{l}5.250 \\
\text { (geplant } \\
\text { ) }\end{array}$ & N/A & $\begin{array}{l}\text { NYHA II; LVEF } \\
>40 \% \text { und NT- } \\
\text { proBNP } \geq 300 \\
\text { pg/ml; Alter } \geq 18 \\
\text { Jahre }\end{array}$ & $\begin{array}{l}\text { eGFR }<20 \\
\mathrm{ml} / \mathrm{min} / 1,73 \mathrm{~m}^{2}\end{array}$ \\
\hline $\begin{array}{l}\text { EMPEROR } \\
\text {-Reduced }\end{array}$ & N/A & $\begin{array}{l}\text { Empagliflozi } \\
\mathrm{n}\end{array}$ & $\begin{array}{l}3.600 \\
\text { (geplant } \\
\text { ) }\end{array}$ & N/A & $\begin{array}{l}\text { LVEF } 36-40 \% \text { und } \\
\text { NT-proBNP } \geq 2500 \\
\text { pg/ml; LVEF } 31- \\
35 \% \text { und NT- } \\
\text { proBNP } \geq 1000 \\
\text { pg/ml; LVEF } \leq 30 \% \\
\text { und NT-proBNP } \\
\geq 600 \text { pg/ml; Alter } \\
\geq 18 \text { Jahre }\end{array}$ & $\begin{array}{l}\text { eGFR }<20 \\
\mathrm{ml} / \mathrm{min} / 1,73 \mathrm{~m}^{2}\end{array}$ \\
\hline
\end{tabular}




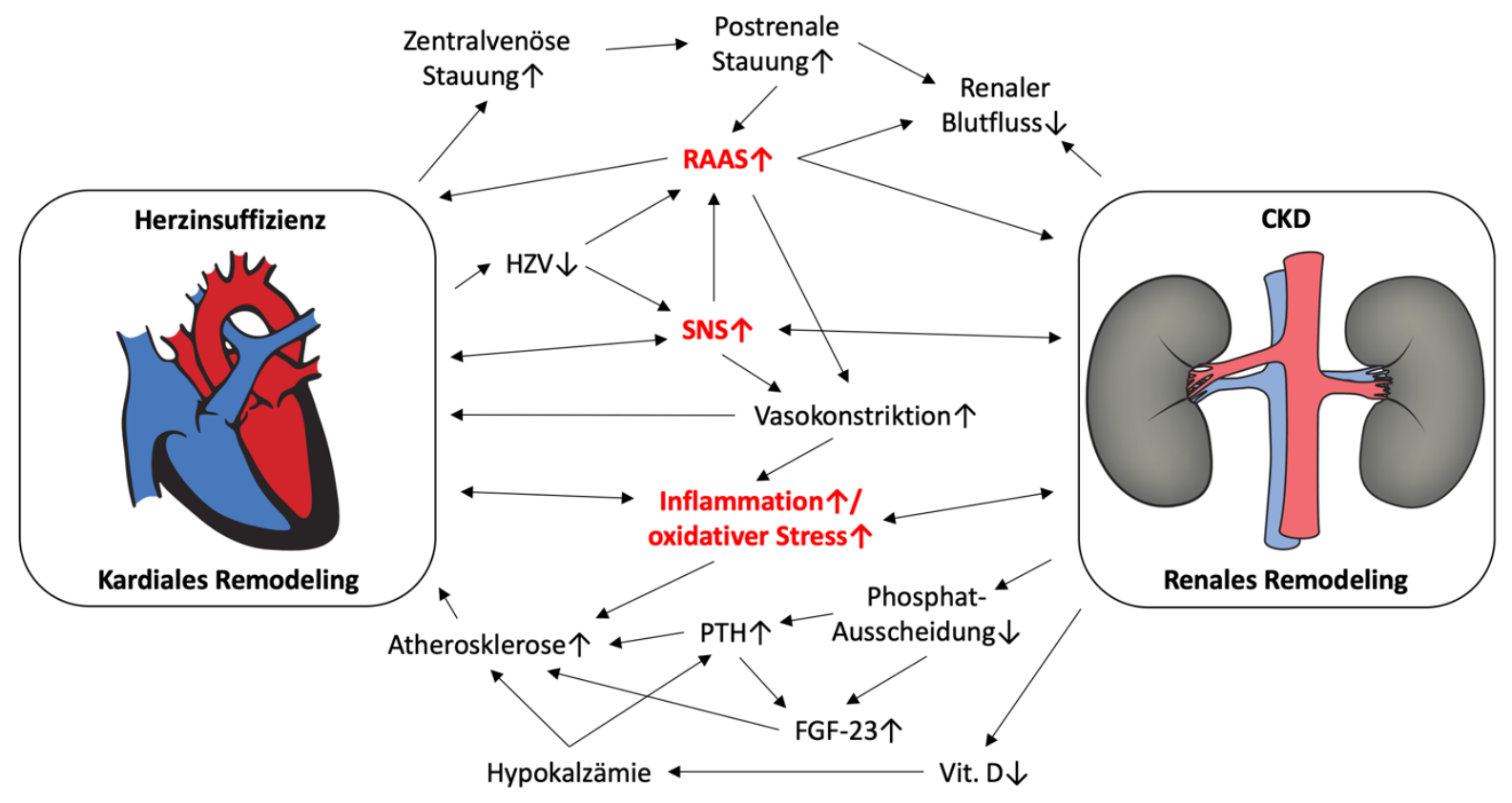




\begin{tabular}{|c|c|c|c|c|c|c|}
\hline & & & & Albun & urie (ACF & $\mathrm{mg} / \mathrm{g}$ ) \\
\hline & & & & $\mathbf{A 1}$ & $\mathbf{A 2}$ & A3 \\
\hline & & & & $<30$ & $30-300$ & $>300$ \\
\hline$\sqrt{2}$ & G1 & Hoch/normal & $>90$ & & & \\
\hline$m$ & G2 & Leicht verringert & $60-89$ & & & \\
\hline$\stackrel{1}{\varepsilon}$ & G3a & Leicht bis moderat verringert & 45-59 & & & \\
\hline ह & G3b & Moderat bis stark verringert & $30-44$ & & & \\
\hline$\frac{\varepsilon}{\alpha}$ & G4 & Stark verringert & $15-29$ & & & \\
\hline ৫ & G5 & Nierenversagen & $<15$ & & & \\
\hline
\end{tabular}

*Albumin-Kreatinin-Quotient 


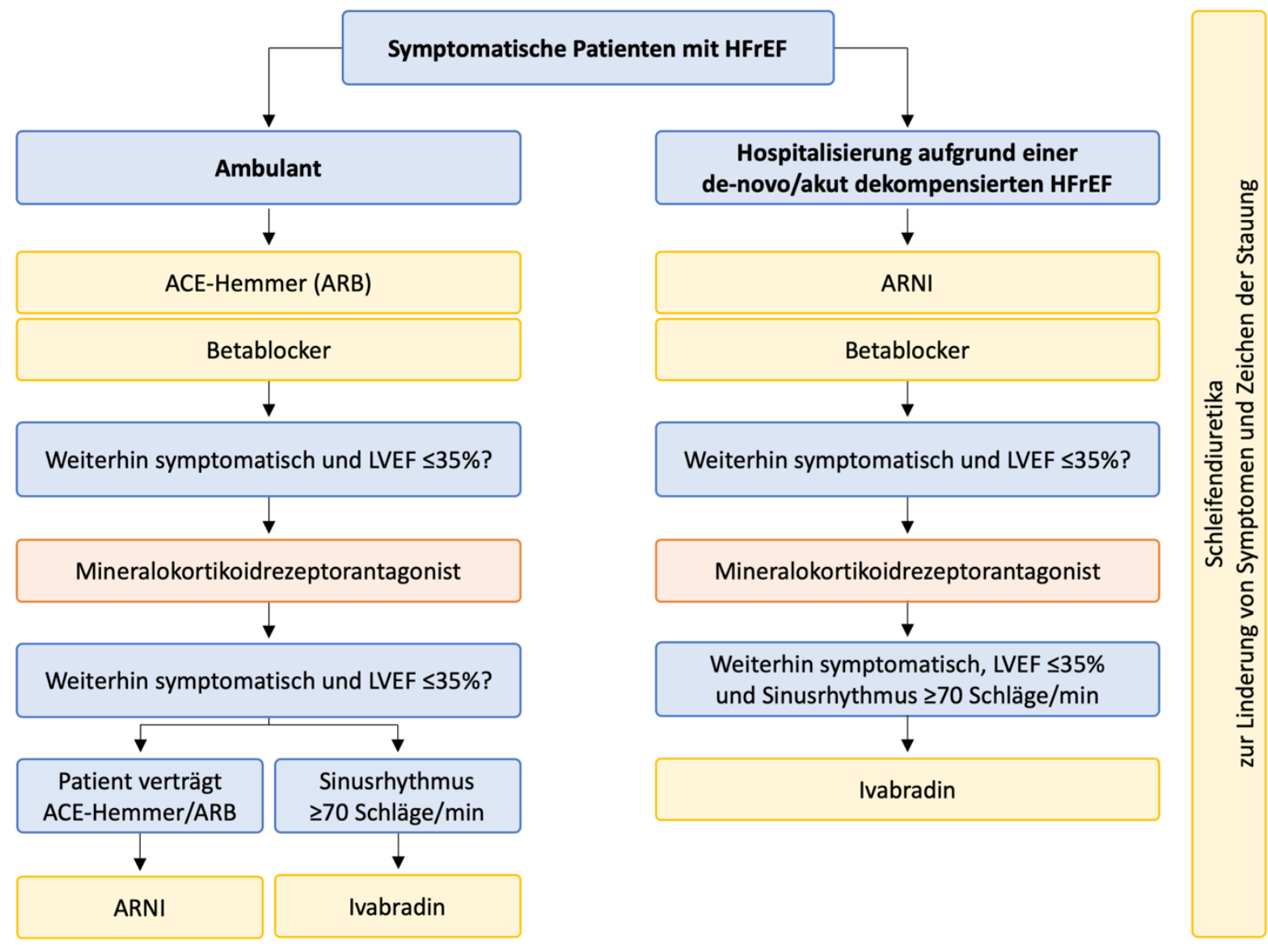

Besonderheiten bei einer eGFR $<30 \mathrm{~mL} / \mathrm{min} / 1,73 \mathrm{~m}^{2}$

Nur unter besonderer Vorsicht

Kontraindiziert 